\title{
Remote Effects of Focal Hippocampal Seizures on the Rat Neocortex
}

\author{
Dario J. Englot, ${ }^{1}$ Asht M. Mishra, ${ }^{1}$ Peter K. Mansuripur, ${ }^{1}$ Peter Herman, ${ }^{2,6,7}$ Fahmeed Hyder, ${ }^{2,3,6,7}$ and \\ Hal Blumenfeld ${ }^{1,4,5,6}$ \\ Departments of ${ }^{1}$ Neurology, ${ }^{2}$ Diagnostic Radiology, ${ }^{3}$ Biomedical Engineering, ${ }^{4}$ Neurobiology, and ${ }^{5}$ Neurosurgery, ${ }^{6}$ Program for Quantitative Neuroscience \\ with Magnetic Resonance (QNMR), and ${ }^{7}$ Magnetic Resonance Research Center (MRRC), Yale University School of Medicine, New Haven, Connecticut 06520
}

Seizures have both local and remote effects on nervous system function. Whereas propagated seizures are known to disrupt cerebral activity, little work has been done on remote network effects of seizures that do not propagate. Human focal temporal lobe seizures demonstrate remote changes including slow waves on electroencephalography (EEG) and decreased cerebral blood flow (CBF) in the neocortex. Ictal neocortical slow waves have been interpreted as seizure propagation; however, we hypothesize that they reflect a depressed cortical state resembling sleep or coma. To investigate this hypothesis, we performed multimodal studies of partial and secondarily generalized limbic seizures in rats. Video/EEG monitoring of spontaneous seizures revealed slow waves in the frontal cortex during behaviorally mild partial seizures, contrasted with fast polyspike activity during convulsive generalized seizures. Seizures induced by hippocampal stimulation produced a similar pattern, and were used to perform functional magnetic resonance imaging weighted for blood oxygenation and blood volume, demonstrating increased signals in hippocampus, thalamus and septum, but decreases in orbitofrontal, cingulate, and retrosplenial cortex during partial seizures, and increases in all of these regions during propagated seizures. Combining these results with neuronal recordings and CBF measurements, we related neocortical slow waves to reduced neuronal activity and cerebral metabolism during partial seizures, but found increased neuronal activity and metabolism during propagated seizures. These findings suggest that ictal neocortical slow waves represent an altered cortical state of depressed function, not propagated seizure activity. This remote effect of partial seizures may cause impaired cerebral functions, including loss of consciousness.

Key words: consciousness; BOLD decreases; cortex; fMRI; slow oscillations; temporal lobe epilepsy

\section{Introduction}

The nervous system performs highly efficient computations by using both local circuits and long-range network interactions. Seizures disrupt this normal brain function on multiple levels. In partial seizures, there is massive synchronized neuronal firing and impaired function in a local region of the brain. If a partial seizure propagates, or secondarily generalizes, then remote brain regions can also be disrupted by abnormally intense neuronal firing. What happens to the function of remote regions during a partial seizure that does not propagate from its region of onset? There are numerous examples where increased activity in one part of the cerebral cortex causes an inhibitory surround, such as in normal visual or somatosensory processing (McCasland et al., 1991; Brumberg et al., 1996; Angelucci et al., 2002), or abnormal surround inhibition in focal cortical seizures (Prince and Wilder, 1967; Collins, 1978; Schwartz and Bonhoeffer, 2001). However,

Received May 1, 2008; revised July 1, 2008; accepted July 31, 2008.

This work was supported by National Institutes of Health Grants R01 NS049307 (H.B.), F30 NS59074 (D.J.E.), and P30 NS052519 and R01 MH067528 (F.H.), and by the Betsy and Jonathan Blattmachr family. We thank M. Purcaro, J. Motelow, and M. Enev for computer programming; U. Schridde and B. Ganganna for technical advice; and D. Ellens and J. Brumberg for helpful comments on this manuscript. We also thank T. Eid, Y. Wang, H. Zaveri, and N. de Lanerolle for the use of recording equipment during pilot studies.

Correspondence should be addressed to Dr. Hal Blumenfeld, Department of Neurology, Yale University, 333 Cedar Street, New Haven, CT 06520. E-mail: hal.blumenfeld@yale.edu.

D01:10.1523/JNEUROSCI.2014-08.2008

Copyright $\odot 2008$ Society for Neuroscience $\quad$ 0270-6474/08/289066-16\$15.00/0 the ability of focal hippocampal seizures to produce long-range depressive effects on the neocortex has not been studied in detail.

Temporal lobe epilepsy (TLE) is a common and debilitating neurological disorder, characterized by seizures that often originate from limbic structures, including the hippocampus (Engel, 1987; Williamson et al., 1993). Interestingly, partial temporal lobe seizures often cause functional deficits beyond those expected from local hippocampal impairment. Thus, in addition to amnesia, patients typically exhibit repetitive automaton-like movements and profoundly impaired consciousness during and after partial temporal lobe seizures. It has been proposed that even when temporal lobe seizures do not propagate, they may cause remote dysfunction in other regions, leading to these deficits (Van Paesschen et al., 2003; Blumenfeld et al., 2004a,b). Indeed, several neuroimaging studies using single-photon emission-computed tomography (SPECT) have revealed increased cerebral blood flow (CBF) associated with epileptic activity in the temporal lobe, but simultaneous decreased CBF in frontoparietal neocortex (Rabinowicz et al., 1997; Menzel et al., 1998; Chang et al., 2002). In a recent SPECT study, we reported that frontoparietal decreases in CBF during temporal lobe seizures in humans were correlated with deficits in consciousness (Blumenfeld et al., 2004a). We also found that these same frontoparietal regions exhibit large-amplitude $1-2 \mathrm{~Hz}$ slow waves on intracranial electroencephalography (EEG), most prominent in the or- 
bitofrontal cortex (Blumenfeld et al., 2004b). The frontoparietal CBF decreases and slow waves occur both during (ictal) and after (postictal) seizures, when consciousness is impaired. Do these neocortical changes represent seizure propagation, or a distinct state of depressed cerebral function more closely resembling coma, deep anesthesia, or sleep (Steriade et al., 1993; Cowan and Wilson, 1994; Haider et al., 2006)?

To directly investigate this question, we performed multimodal studies of limbic seizures in rats. We observed neocortical slow waves associated with reduced neuronal activity and cerebral metabolism during partial limbic seizures, contrasted with increased neuronal activity and metabolism in the neocortex during seizure propagation. Our findings suggest that ictal neocortical slow waves represent an altered cortical state of depressed function, not propagated seizure activity, which may play an important role in impaired cerebral function during partial seizures.

\section{Materials and Methods}

\section{Animal preparation and surgery}

All procedures were in full compliance with approved institutional animal care and use protocols. A total of 62 adult male and female Sprague Dawley rats (Charles River Laboratories) weighing 190-460 gm were used in these experiments. Fourteen animals were used for awakebehaving seizure monitoring after pilocarpine administration, 29 were used for acute electrophysiology and laser Doppler flowmetry (LDF) experiments, and 19 were used for multimodal functional magnetic resonance imaging (fMRI) studies.

Awake-behaving seizure experiments. At least $3 \mathrm{~d}$ before continuous video/EEG seizure monitoring, animals were surgically anesthetized with ketamine $(100 \mathrm{mg} / \mathrm{kg})$, xylazine $(10 \mathrm{mg} / \mathrm{kg})$, and acepromazine $(1 \mathrm{mg} / \mathrm{kg}$, i.m., q1 h) and stereotactically implanted with a bipolar Teflon coated stainless steel recording electrode $(50-100 \mathrm{k} \Omega$ resistance, Plastics One) in each of two regions: the dorsal hippocampus [anteroposterior (AP), -3.8 ; mediolateral $(\mathrm{ML}),+2.5$; superior-inferior $(\mathrm{SI}),-2.6]$ and the lateral orbitofrontal cortex $(\mathrm{AP},+4.2 ; \mathrm{ML},+2.2$; SI, -2.4$)$. All coordinates are in millimeters relative to bregma (Paxinos and Watson, 1998). A screw in the midline skull behind lambda served as ground and recording reference.

Acute electrophysiology/CBF experiments. Before acute electrophysiology experiments, animals were anesthetized with ketamine/xylazine (90/15 mg/kg, i.m.). Responsiveness was checked every $15 \mathrm{~min}$ by toe pinch. A high-impedance recording microelectrode (2-4 $\mathrm{M} \Omega$ resistance; FHC) was fixed to a bipolar Teflon coated stainless-steel electrode (Plastics One) and stereotactically placed in the dorsal hippocampus, while a second high-impedance microelectrode was placed in the orbitofrontal cortex (same coordinates as above). During most experiments, an OxyFlo XP needle probe (Oxford Optronix) was also fixed to each electrode to measure CBF. A screw in the midline skull behind lambda served as ground and recording reference. At the conclusion of both chronic and acute experiments, animals were killed with an intraperitoneal injection of Euthasol (Virbac Animal Health) and brains were collected to histologically verify electrode locations.

Acute fMRI experiments. At least $6 \mathrm{~d}$ before fMRI recordings, animals were stereotactically implanted with a MRI-compatible bipolar tungsten stimulating/recording electrode $(\sim 0.1 \mathrm{M} \Omega$ resistance; Microprobe) in the dorsal hippocampus (same coordinates as above). To minimize hardware and susceptibility artifact in the vicinity of the fMRI measurements, the electrode was lowered at a $50^{\circ}$ angle from the vertical using a posterior approach and fixed to the skull adjacent to lambda with two to four plastic screws and dental acrylic. Immediately before fMRI scans, animals were anesthetized with ketamine/xylazine ( $90 / 15 \mathrm{mg} / \mathrm{kg}$, i.m., q1 h), tracheotomized, and artificially ventilated $\left(70 \%\right.$ air and $\left.30 \% \mathrm{O}_{2}\right)$. To prevent any movements during the experiments and to facilitate artificial breathing, animals were paralyzed by repeated injections of D-tubocurarine chloride $(0.5 \mathrm{mg} / \mathrm{kg}$, i.v.) (Sigma-Aldrich). A femoral artery was cannulated (Intramedic PE50 tubing; Becton Dickinson) for continuous arterial blood pressure monitoring and periodic blood sam- pling for measurements of blood gases and $\mathrm{pH}$ using an ABL 5 blood gas analyzer (Radiometer Copenhagen). All physiological values (blood gases, mean arterial blood pressure, and $\mathrm{pH}$ ) were maintained within physiological range throughout the experiments (Schridde et al., 2008). One femoral vein was cannulated and an IP line was placed (Intramedic PE10 tubing) for the injections of anesthetics and paralyzing agents. For flow-related cerebral blood volume (CBV) fMRI experiments, rats were also injected with iron oxide contrast agent $(24-50 \mathrm{mg} / \mathrm{kg}$ ) [Combidex (ferumoxtran-10); Advanced Magnetics). Body temperature was kept constant at $\sim 37^{\circ} \mathrm{C}$ using a heating pad.

\section{Seizure induction and recording procedures}

Awake-behaving seizure experiments. We used the pilocarpine rat model of TLE (Turski et al., 1983; Cavalheiro et al., 1991) for awake-behaving experiments. In this model, animals develop spontaneous limbic seizures within days to weeks of drug-induced status epilepticus, often displaying behaviorally mild seizures at first, which progress into generalized tonicclonic seizures over time (Turski, 2000). Status epilepticus was induced at least $1 \mathrm{~d}$ before surgical implant. Animals were pretreated with scopolamine methyl nitrate $(1 \mathrm{mg} / \mathrm{kg}$, s.c. $)$ to antagonize peripheral effects induced by subsequent pilocarpine ( $405 \mathrm{mg} / \mathrm{kg}$, s.c.), injected $30 \mathrm{~min}$ later. Although injections induced status epilepticus (i.e., sustained seizures lasting $>30 \mathrm{~min}$ ) within 10-30 min in most animals, animals not entering status received up to two supplemental doses of pilocarpine ( $\sim 20 \%$ full dose) at $\sim 45$ min intervals. One hour after the onset of status epilepticus, diazepam $(7.5 \mathrm{mg} / \mathrm{kg}$, s.c. $)$ and saline $(5 \mathrm{ml}$, s.c. $)$ were administered to limit further seizure activity and allow rehydration. Animals were checked $\sim 3$ and $5 \mathrm{~h}$ later, and if signs of behavioral seizure activity still persisted, supplemental doses of diazepam and saline $(\sim 50 \%$ full dose) were administered.

Four to six days after status epilepticus, implanted animals were placed under $24 \mathrm{~h}$ video/EEG monitoring in custom Plexiglas cages which allowed constant access to food and water. Local field potential (LFP) signals were recorded from frontal and hippocampal electrodes referenced to ground using steel-coated EEG cables and swivel commutators (Plastics One) and a model 12 amplifier (Grass Instruments). LFP signals were filtered $0.3-100 \mathrm{~Hz}$, amplified $\times 500$, and digitized with a Power 1401 (Cambridge Electronic Design) at a sampling rate of $1 \mathrm{kHz}$. Video recordings of behavior were obtained with a BD400 camera (Panasonic) and captured with a VC-211A analog-to-digital video converter (Actionstar). Two hour segments of $24 \mathrm{~h}$ simultaneous electrophysiology and video data were collected continuously using Spike 2 software (Cambridge Electronic Design) and were reviewed daily for seizures. It was observed that seizures typically fit into one of two electrographic categories based on frontal LFP recordings. The first category included seizures associated with large-amplitude $1-2 \mathrm{~Hz}$ slow activity in the frontal cortex, which contrasted starkly with $9-12 \mathrm{~Hz}$ fast polyspike seizure activity observed in the hippocampus. These were defined as "partial" seizures with ictal neocortical slow waves. The second category consisted of seizures associated with $9-12 \mathrm{~Hz}$ fast polyspike activity in the frontal cortex, which was synchronized with fast seizure activity in the hippocampus. These were defined as propagated or "secondarily generalized" seizures. Although some seizures did contain some epochs of both slow and fast seizure activity in the frontal cortex, one rhythm clearly predominated (for more than two-thirds of the seizure duration) throughout most seizures. Using these criteria, electrographic seizure classification was assigned by two independent raters blinded to behavioral seizure severity. Video data during seizures were used to assess behavioral seizure severity based on the Racine (1972) scale ranging from class $0-5$. Because animal position on video did not always allow discernment of the subtle differences between class 0 seizures (behavioral arrest/staring without facial automatisms) and class 1 seizures (behavioral arrest/staring with facial automatisms such as chewing), these two seizure classes were combined into one category. Animals were recorded from continuously and then removed from the monitoring system $21 \mathrm{~d}$ after pilocarpine injection, or if five consecutive secondarily generalized seizures were recorded, or if LFP signal quality became poor in either region.

Acute electrophysiology/CBF experiments. After electrode placement, 2 min of recordings were collected under deep ketamine/xylazine anesthe- 
sia using a Microelectrode AC Amplifier (model 1800; A-M Systems), broad-band filtered from $0.1 \mathrm{~Hz}$ to $10 \mathrm{kHz}$ ( $\times 100$ gain). Signals were then filtered with a model 3363 filter (Krohn-Hite) into LFPs (0.1-100 $\mathrm{Hz}$ ) and multiunit activity (MUA) $(400 \mathrm{~Hz}$ to $10 \mathrm{kHz}$ ) (Shmuel et al., 2006; Schridde et al., 2008). For CBF-measurement experiments, we used a model 4000 LDF system (Oxford Optronix). Electrophysiology and CBF signals were digitized with a Power 1401 (Cambridge Electronic Design) at a sampling rate of $1 \mathrm{kHz}$ for LFPs and CBF and $20 \mathrm{kHz}$ for MUA, and recorded using Spike 2 software. After recordings under deep anesthesia, no further anesthetic was administered for 30-60 min before seizure stimulation to allow the animal to enter a lightly anesthetized state. Animals were defined as "lightly anesthetized" when the frequency of large, positively deflected delta oscillations on frontal cortex LFPs decreased to less than three per $10 \mathrm{~s}$ of recordings, compared with a frequency of $\sim 12-15$ delta waves per $10 \mathrm{~s}$ under deep anesthesia (Maandag et al., 2007). A recent study of ketamine/xylazine anesthesia in rats noted similar reduction of delta waves, as well as increased heart rate and respiratory rate, at the transition from deep to light anesthesia, suggesting that suppression of LFP delta activity provides a reliable prediction of the lightly anesthetized state (Musizza et al., 2007). In our lightly anesthetized state, the animals' respiratory and heart rates typically became elevated $\sim 50 \%$ above the rate under deep anesthesia, but the animal remained unresponsive to somatosensory stimulation.

To induce a seizure during the lightly anesthetized state, a $2 \mathrm{~s}$ stimulus train was delivered between the two hippocampal stimulating electrodes consisting of square biphasic ( $1 \mathrm{~ms}$ each phase) pulses at $60 \mathrm{~Hz}$, using an Isolated Pulse Stimulator (model 2100; A-M Systems). The current intensity of the stimulus differed depending on whether we intended to record partial seizures, in which fast seizure activity did not propagate to the frontal cortex, or secondarily generalized seizures, in which fast seizure activity did propagate to the frontal cortex. We observed during pilot experiments that, whereas stimuli $<1500 \mu$ A typically created partial seizures, those of higher currents typically induced secondarily generalized seizures. Thus, during experiments investigating partial seizures, the current was titrated by $\sim 200 \mu \mathrm{A}$ steps to the lowest stimulus intensity (typically $200-800 \mu \mathrm{A}$ ) that would produce a seizure $>45 \mathrm{~s}$ duration in the hippocampus. Partial seizure stimuli did not exceed $1500 \mu \mathrm{A}$. Between subthreshold stimuli, we allowed a 15 min rest period and administered a $20-30 \%$ supplemental dose of anesthesia if the animal became responsive to toe pinch. In contrast, during experiments investigating secondarily generalizing seizures, the intensity of the stimuli began at 2 $\mathrm{mA}$. In addition, although there was no stimulus titration in this latter type of experiment, at least $1 \mathrm{~h}$ before experiments recording secondarily generalized seizures, a preliminary seizure was induced using a $2 \mathrm{~mA}$ stimulus because it was found that this helped to "kindle" the hippocampus and make secondary generalization during later seizures more likely.

In both types of experiments, after the first seizure was recorded, the animal was allowed to recover from the postictal state back to the lightly anesthetized state, and then anesthetic was again administered. After at least $15 \mathrm{~min}$, another $2 \mathrm{~min}$ of deep anesthesia recordings were collected, and the entire protocol was repeated. The experiment was terminated if up to three usable seizures were recorded or if a stimulus did not result in a seizure $>45 \mathrm{~s}$.

Acute fMRI experiments. After surgery and preparation for fMRI experiments, animals were switched from high-dose ketamine/xylazine $(90 / 15, \mathrm{mg} / \mathrm{kg})$ to a low-dose $(40 / 7, \mathrm{mg} / \mathrm{kg})$, and maintained under light anesthesia using this lower dose throughout the experiment. Two types of experiments were performed: those recording partial seizures and those recording secondarily generalized seizures, using the same stimulation protocols as defined in the preceding section. One to four stimulation experiments were performed in each animal, with at least $20 \mathrm{~min}$ between stimuli.

During MRI recordings, the rat was positioned prone in a specially designed plastic holder with the head fixed and bregma positioned at the center of the surface coil. The animal was then inserted into the magnet with its head positioned at the magnetic isocenter. To record simultaneous MRI and EEG signals while minimizing MRI signal distortion, a pair of carbon-filament electrodes (diameter, $1 \mathrm{~mm}$; WPI) were placed laterally between the scalp and the outside surface of the skull in the left frontal and occipital areas, secured to the skin with tissue glue (Nersesyan et al., 2004). To minimize mechanical transmission of acoustic vibrations from the MRI scanner which can cause artifacts in the EEG signal, the wires were fixed to the lateral surface of the holder and bound together using plastic adhesive tape. The EEG signals were amplified $(\times 100)$ and filtered $(1-100 \mathrm{~Hz})$ using a model 79D data recording system (Grass Instruments). In addition, hippocampal LFPs were recorded simultaneously with fMRI using bipolar tungsten electrodes connected to a double copper wire (Microprobe) bound to the holder with plastic tape, and filtered $1-100 \mathrm{~Hz}(\times 100)$ with a Microelectrode AC Amplifier (model 1800; A-M Systems). EEG and LFP signals were digitized and recorded (sampling rate $1000 \mathrm{~Hz}$ ) using a Cambridge Electronic Design Micro 1401 and Spike 2 software.

All MRI experiments were acquired on a 9.4 tesla Bruker horizontal bore (16 $\mathrm{cm}$ bore diameter) spectrometer equipped with passively shielded shim/gradient coils operating at $400.5 \mathrm{MHz}$ for ${ }^{1} \mathrm{H}$. The transceiver system consisted of a ${ }^{1} \mathrm{H}$ resonator radio frequency surface coil (15 $\mathrm{mm}$ diameter) for transmission of radio frequency pulses and receiving. To optimize the homogeneity of the static magnetic field, the system was shimmed before each experiment using global automated shimming (Gruetter, 1993). High spatial resolution anatomical images for each animal were acquired with 10 interlaced slices in the coronal plane using the fast low-angle shot sequence with a time repetition (TR) of $500 \mathrm{~ms}$, an time echo (TE) of $6 \mathrm{~ms}$, a flip angle of $40-55^{\circ}$, field of view (FOV) of $25 \times$ $25 \mathrm{~mm}$, a $256 \times 256$ matrix, an in-plane resolution of $98 \times 98 \mu \mathrm{m}$, and $1000 \mu \mathrm{m}$ slice thickness. Blood oxygenation level-dependent (BOLD) and flow-related CBV fMRI data were obtained in the same planes as anatomical images using single-shot spin echo, echo planar imaging with the following parameters: TR, 1000 (or 2000) ms; TE, 25 ms; excitation flip angle, $90^{\circ}$; inversion flip angle, $180^{\circ}$; FOV, $25 \times 25 \mathrm{~mm}$; $64 \times 64$ matrix; in-plane resolution of $390 \times 390 \mu \mathrm{m}$; and slice thickness, 1000 $\mu \mathrm{m}$. The fMRI image acquisitions of 10 slices were obtained over $1 \mathrm{~s}$ (or over $2 \mathrm{~s}$ ) with a total time from one image onset to the next of $3 \mathrm{~s}$. Therefore, with eight dummy scans and 200 repetitions, a total of 2000 images were obtained per $600 \mathrm{~s}$ experiment.

\section{Data analysis and statistics}

Awake-behaving seizure experiments. To analyze the relationship between electrographic seizure propagation and behavioral severity during seizures in pilocarpine-treated animals, we created a $2 \times 2$ contingency table based on two nominal variables: electrographic seizure classification (partial vs secondarily generalized) and behavioral seizure class (nonmotor seizures of Racine class $0-1$ vs convulsive seizures of Racine class 2-5). Seizures were classified as partial or generalized based on EEG by two readers (D.J.E., A.M.M.) who were blinded to the behavioral seizure class. A $\chi^{2}$ test was applied to assess statistical significance. In addition, EEG power spectra were generated for each event using hippocampal and frontal LFP data during four defined states: (1) "baseline" was the last $20 \mathrm{~s}$ of stable awake recordings before each seizure, (2) "seizure" was the first $20 \mathrm{~s}$ of seizure activity (determined from hippocampal LFPs), (3) "postictal" was first $20 \mathrm{~s}$ after the seizure, and (4) "sleep" was defined as a 20 s epoch from the closest episode of slow-wave, nonparadoxical (i.e., non-REM) sleep (identified using video/EEG recordings). Of the two distinct stages of sleep well defined in rats, slowwave sleep is distinguishable as it typically associated with largeamplitude delta waves in several cortical and subcortical structures, whereas paradoxical sleep typically contains fast cortical spindles and elevated hippocampal theta (Gottesmann, 1996; Crisler et al., 2008). All four states were defined for both partial and generalizing seizures. Epochs of "mixed" propagating and partial seizure activity were excluded from analysis of both seizure types. To generate power spectra, all LFP data were subjected to fast Fourier transformation (FFT) analysis using Spike 2. All power amplitudes were normalized to the peak amplitude of the baseline power spectrum (peak power at baseline, 1).

Notably, although both partial and generalized limbic seizures in both stimulated and spontaneously seizing animals were most often characterized by fast large-amplitude 9-12 Hz LFP activity in the hippocampus, other hippocampal spiking patterns were sometimes observed during portions of the seizure. These patterns, including low-voltage fast $(>15$ 
$\mathrm{Hz}$ ) or large-amplitude slow $(<3 \mathrm{~Hz})$ hypersynchronous spiking, have been described previously in limbic epilepsy in both humans and animals (Bragin et al., 1999, 2005; Velasco et al., 2000), and were thus considered part of the seizure and included in all respective analyses.

Acute electrophysiology/CBF experiments. To analyze changes in neuronal activity during acute recordings of electrically stimulated limbic seizures in lightly anesthetized animals, LFP and MUA signal amplitude was also processed using Spike 2 software. To track changes in LFP and MUA signals during both partial and secondarily generalized seizures, the root mean square (RMS) voltage was used as a measure of signal power, calculated in nonoverlapping $5 \mathrm{~s}$ time bins from $100 \mathrm{~s}$ before stimulus delivery to $350 \mathrm{~s}$ after. Mean LDF signals were also calculated in $5 \mathrm{~s}$ bins to track CBF changes. Data time points were synchronized to the time of stimulus onset, and plotted as mean percentage change \pm SEM from a $30 \mathrm{~s}$ uninterrupted baseline recording before the stimulus. We used the RMS amplitude of MUA as an estimate of action potential firing because this has been shown to correspond well to spiking rate measured by template matching (Shmuel et al., 2006; Schridde et al., 2008), and template matching during seizures is unreliable because single units in the hippocampus are replaced by population spikes (Schridde et al., 2008). Data were prepared for analysis by defining four states: (1) "baseline" was the last $45 \mathrm{~s}$ of light-anesthesia recordings before the stimulus, (2) "seizure" represented the first $45 \mathrm{~s}$ after the stimulus, (3) "postictal" was the first $45 \mathrm{~s}$ after the cessation of hippocampal seizure activity, and (4) "deep anesthesia" represented the closest $45 \mathrm{~s}$ deep anesthesia recording (collected at least $15 \mathrm{~min}$ after administering the anesthesia dose). LFP signals during all four states were subjected to FFT analysis in Spike 2 to generate mean power spectra. Mean LFP, MUA, and LDF signal changes during seizure, postictal, and deep anesthesia were each compared with baseline with a paired, two-tailed $t$ test with Bonferroni's correction for multiple comparisons, where applicable. All statistical tests were processed using SPSS 15 (SPSS) or Excel (Microsoft), and significance was assessed at $p \leq 0.05$.

Acute fMRI experiments. EEG and hippocampal LFP signals from fMRI experiments were first processed by applying a low-pass filter at $30 \mathrm{~Hz}$ using Spike 2 software to reduce residual artifacts in the EEG from magnetic field gradients (supplemental Fig. 1, available at www.jneurosci.org as supplemental material). All data were realigned according to individual stimulus time. MRI images were processed using in-house software running on a Matlab platform (MathWorks) written by F. Hyder, M. Enev, J. Motelow, and M. Purcaro (Yale University School of Medicine, New Haven, CT). Although rats were paralyzed during experiments, all fMRI series were first screened for movement artifacts using a MOVIE function and center of mass analysis (restricted to voxels within the brain boundaries) to ensure that all runs exhibited movement of $<25 \%$ of a pixel in either plane of the center of mass image. For BOLD and flowrelated $\mathrm{CBV}$ experiments, $t$-maps were calculated continuously for groups of 10 consecutive image acquisitions (30 s) from stimulus artifact onwards, compared with the same number of baseline image acquisitions immediately preceding the stimulus. A threshold of $t=2$ (corresponding to $p \approx 0.05$ ) was used for $t$-map images, which were then superimposed onto corresponding high-resolution anatomical images for each slice from each individual animal.

For flow-related CBV measurements, an increase in volume causes an increase in the amount of the paramagnetic contrast agent in any given voxel, causing a decrease in T2 and a drop in the fMRI signal. Thus, negative signal changes are associated with increased $\mathrm{CBV}$, and positive signal changes are related to decreased CBV. However, for clarity and better comparison with other signals, we inverted the CBV signal in all analyses and figures, and hence, increases in the signal portray increases in CBV.

To analyze and compare the temporal relationship between BOLD and CBV signal changes measured in the orbitofrontal cortex and hippocampus during fMRI experiments with LFP, MUA, and LDF signal changes recorded in those regions during bench experiments, regions of interest (ROIs) were chosen for the MRI data analysis based on coordinates used for bench experiments. In addition, four other ROIs were analyzed corresponding to regions of consistent and prominent BOLD and CBV changes, including the thalamus, septal nuclei, anterior cingulate cortex, and retrosplenial/posterior cingulate cortex. All ROIs were chosen using high-resolution anatomical images in each animal and a rat brain atlas (supplemental Fig. 2, available at www.jneurosci.org as supplemental material) (Paxinos and Watson, 1998). BOLD and CBV signal changes were calculated as percentage change from the mean of the $30 \mathrm{~s}$ baseline signal before the stimulus for consecutive bins of nonoverlapping $6 \mathrm{~s}$ intervals.

To determine whether signal changes were significantly different between baseline and seizure recordings for all ROIs, the mean percentage signal change for the first $45 \mathrm{~s}$ of seizure activity as well as the mean percentage signal change during an uninterrupted $45 \mathrm{~s}$ baseline recording were calculated. Paired $t$ tests were used to analyze signal changes during seizures compared with baseline recordings, with significance assessed at $p \leq 0.05$. All graphs are plotted as mean \pm SEM over the time range of the experiments in $6 \mathrm{~s}$ bins and synchronized to time of stimulus.

Estimation of $\mathrm{CMRO}_{2}$ changes. Changes in the cerebral metabolic rate of oxygen consumptions $\left(\mathrm{CMRO}_{2}\right)$ during seizures were estimated separately for the hippocampus and orbitofrontal cortex, using the known general relationship (Ogawa et al., 1993; Hyder et al., 2001) between $\mathrm{CMRO}_{2}$ and BOLD, CBV, and CBF at steady state as described as follows:

$\Delta S / S=\grave{A} \times\left[\left(\Delta \mathrm{CBF} / \mathrm{CBF}-\Delta \mathrm{CMRO}_{2} / \mathrm{CMRO}_{2}\right) /\right.$

$$
(1+\Delta \mathrm{CBF} / \mathrm{CBF})-\Delta \mathrm{CBV} / \mathrm{CBV}]
$$

where $\Delta S / S$ represents changes in the BOLD signal relative to baseline, $A$ represents a BOLD calibration parameter, and $\triangle \mathrm{CBF} / \mathrm{CBF}, \Delta \mathrm{CBV} / \mathrm{CBV}$, and $\triangle \mathrm{CMRO}_{2} / \mathrm{CMRO}_{2}$ are changes in $\mathrm{CBF}, \mathrm{CBV}$, and $\mathrm{CMRO}_{2}$, respectively (Hyder et al., 2002). As was shown previously (Schridde et al., 2008), initial analyses with the present data again showed that $\mathrm{CMRO}_{2}$ estimates did not differ appreciably using $\grave{A}$ values of $0.4,0.5$, and 0.6 (data not shown). Therefore, a value of $\grave{A}=0.5$ was used for all final calculations. Details regarding the use of $\mathrm{CMRO}_{2}$ estimates to analyze changes in specific ROIs during fMRI recordings of seizures have been described in detail previously (Schridde et al., 2008).

To calculate the time course of $\Delta \mathrm{CMRO}_{2} / \mathrm{CMRO}_{2}(t)$, we used values from individual experiments for $\Delta S / S(t)$, but we used the average values of $\Delta \mathrm{CBV} / \mathrm{CBV}(t)$ and $\Delta \mathrm{CBF} / \mathrm{CBF}(t)$. We used averages instead of individual values for $\Delta \mathrm{CBV} / \mathrm{CBV}(t)$ and $\Delta \mathrm{CBF} / \mathrm{CBF}(t)$ because we did not acquire CBV measurements in all experiments and performed separate measurements of CBF using LDF instead of arterial spin labeling (ASL) MRI. CBF measurements using LDF have been shown to be comparable with CBF acquired using ASL MRI (Mandeville et al., 1999; He et al., 2007). Furthermore, CBV measured by LDF and MRI demonstrates similar kinetics of red blood cell and blood plasma compartments (Herman et al., 2008). Thus, our measured changes in CBF and CBV are in line with previous observations (Nersesyan et al., 2004; Kida et al., 2007; Schridde et al., 2008). To calculate uncertainties for our $\mathrm{CMRO}_{2}$ estimates, we propagated errors in of $\Delta \mathrm{CMRO}_{2} / \mathrm{CMRO}_{2}(t)$ using partial derivatives. The equations for these calculations have been provided and described in detail previously (Schridde et al., 2008).

\section{Results}

\section{Spontaneous partial limbic seizures are associated with ictal neocortical slow activity}

We first examined spontaneous limbic seizures in 14 rats after pilocarpine-induced status epilepticus using $24 \mathrm{~h}$ video/EEG monitoring. Although all seizures were associated with fast polyspike activity in the hippocampus, typically at $9-12 \mathrm{~Hz}$ frequency, one of two distinct rhythms was present in the frontal cortex during seizures (Fig. 1). Twelve seizures in seven animals displayed mostly large-amplitude $1-2 \mathrm{~Hz}$ slow oscillations in the frontal cortex without propagation of fast spike activity and were thus classified as partial seizures (Fig. $1 \mathrm{~A}$ ). Ictal neocortical slow waves present during partial seizures closely resembled largeamplitude slow oscillations seen during slow-wave sleep (Fig. $1 A$, bottom right). In contrast, 37 seizures in 11 animals showed propagation of fast $9-12 \mathrm{~Hz}$ polyspike activity and were classified 


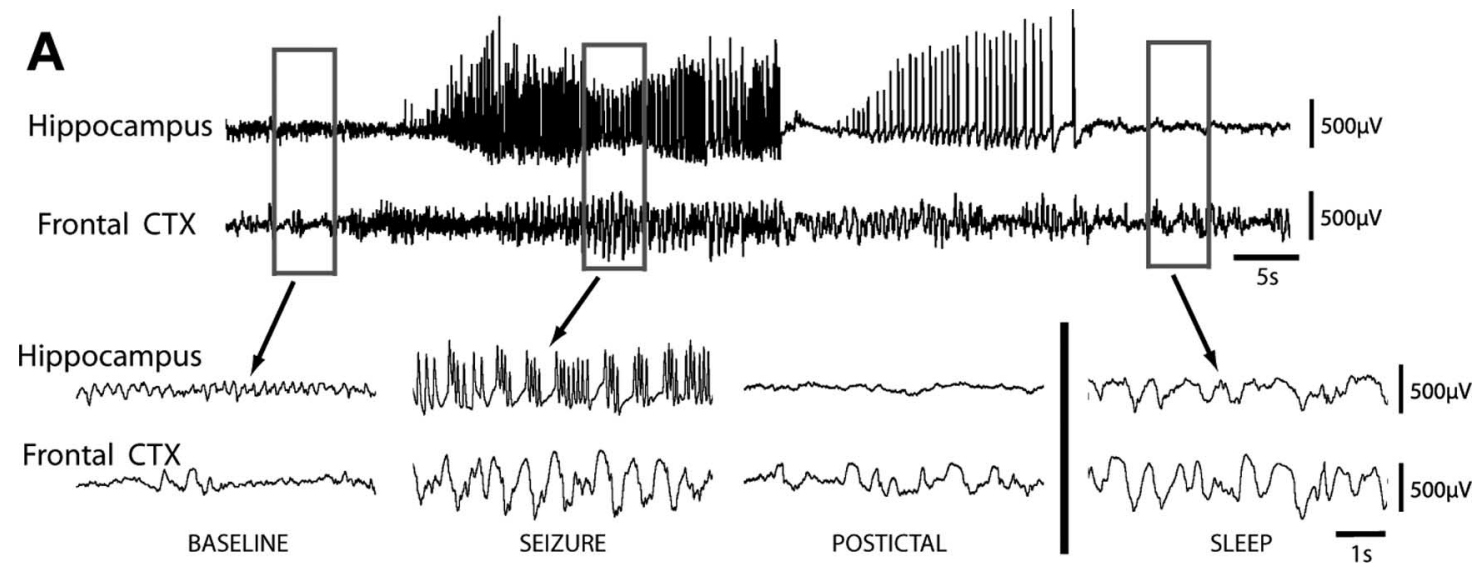

B

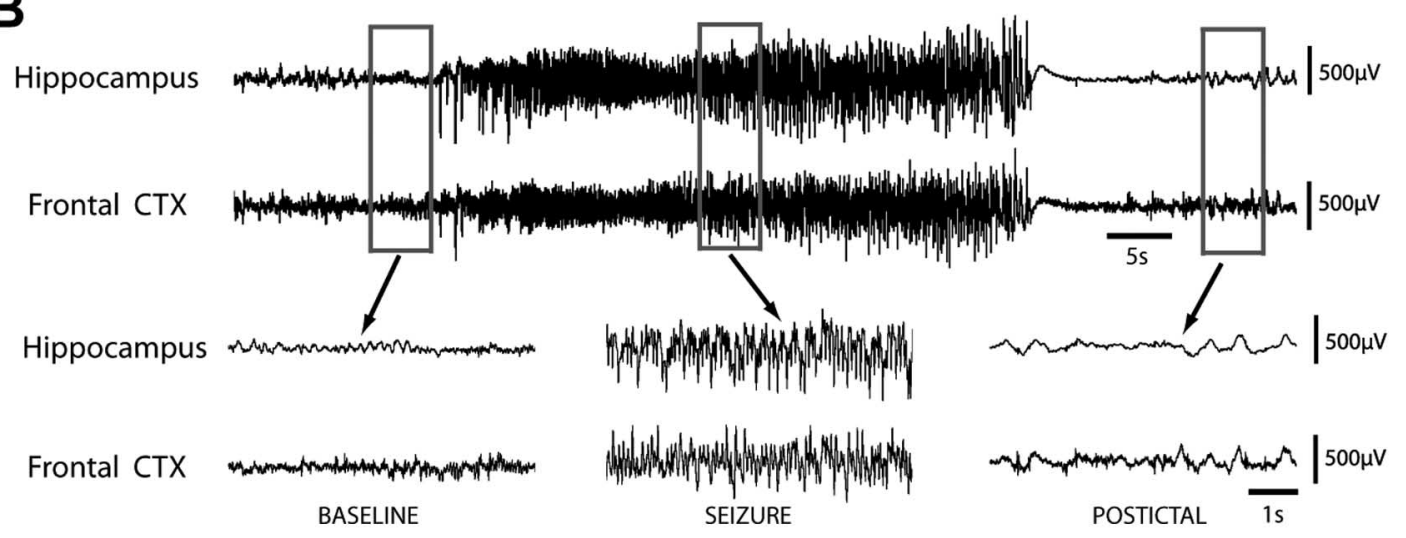

Figure 1. Partial limbic seizures in pilocarpine treated rats are associated with fast polyspike activity in the hippocampus and ictal neocortical slow activity in the frontal cortex. Spontaneous seizures are shown from chronic video/EEG recordings in rats with pilocarpine-induced epilepsy. A, LFPs in the hippocampus and orbitofrontal cortex (CTX) during a Racine class 0 partial seizure of 50 s length, associated with behavioral arrest but no convulsive activity. Hippocampal recordings reveal large-amplitude, fast polyspike activity during the seizure, whereas frontal recordings show large-amplitude 1-2 Hz slow waves during and after the seizure without considerable propagation of fast spike activity. Ictal and postictal slow activity resembles large-amplitude slow rhythms seen during an episode of natural sleep, recorded in the same animal at a different time (bottom, right). B. Hippocampal and frontal recordings during a $46 \mathrm{~s}$ Racine class 5 secondarily generalized seizure, associated with bilateral tonic-clonic convulsions and loss of balance. LFP recordings reveal large-amplitude, fast polyspike activity in both the hippocampus and frontal cortex during the seizure, without frontal slow oscillations. LFP recordings are filtered $0.3-100 \mathrm{~Hz}$.

as secondarily generalized (Fig. 1B). Electrographic classifications between two blinded raters were in agreement for 49 of 52 seizures recorded; the remaining three seizures were deemed by one rater to exhibit mixed slow and propagating frontal activity (neither rhythm predominating more than two-thirds of seizure duration) and were thus excluded. It was noted that whereas partial seizures typically occurred within a few weeks after status epilepticus, often as an animal's first or second recorded seizure, secondarily generalized seizures were more likely to occur after more time had elapsed and after previous seizures had occurred (data not shown). Both partial seizures and secondarily generalized seizures had similar durations at $43.1 \pm 12 \mathrm{~s}$ and $47.6 \pm 16 \mathrm{~s}$ (mean \pm SEM), respectively $(p>0.3)$.

To further characterize EEG rhythms during both seizure types and compare them to sleep rhythms, power spectra were generated for LFP signals during each seizure, its corresponding baseline and postictal recordings, and for the nearest episode of slow-wave sleep (Fig. 2). During partial seizures, hippocampal power increased dramatically across the spectrum examined $(0.3-35 \mathrm{~Hz})$, with the largest peak in the alpha $(8-12 \mathrm{~Hz})$ range, and other peaks in the delta $(0.3-4 \mathrm{~Hz})$ and beta $(12-35 \mathrm{~Hz})$ ranges (Fig. $2 A$ ). In contrast, frontal power increased greatly in the delta range at $1-3 \mathrm{~Hz}$, without higher frequency peaks. This increase in frontal delta power during ictal neocortical slow ac- tivity resembled spectral changes seen in both regions during sleep (Fig. $2 A$, bottom row). During secondarily generalized seizures, considerable power increases were seen in LFPs from both regions, with a large peak at $9-12 \mathrm{~Hz}$ in each area (Fig. $2 \mathrm{~B}$ ). Thus, the pattern of activity in frontal cortex during partial seizure, but not during generalized seizures, resembles cortical activity observed during slow-wave sleep.

To examine a potential relationship between electrographic seizure classification and behavioral manifestations, we rated behavior during all seizures using the Racine scale (Racine, 1972). On this scale, ratings range from the mildest seizures associated only with behavioral arrest (class 0 ) or facial automatisms (class 1) to more severe seizures characterized by head nodding (class 2), bilateral forelimb clonus (class 3), clonus with rearing (class 4), or clonus with loss of balance (class 5) (Racine, 1972). Although 11 of 12 electrographically partial seizures were assigned nonmotor behavioral seizure ratings (i.e., class $0 / 1$ ), all electrographically generalized seizures were associated with more severe behavioral convulsions (class 2-5) (Table 1). A $2 \times 2$ contingency table analysis of partial versus generalized seizures and nonmotor (class $0 / 1$ ) versus convulsive (class 2-5) seizures revealed that this difference was statistically significant $\left(\chi^{2}=43.7\right.$; $p<0.0001$ ). A more conservative $2 \times 2$ contingency analysis of partial versus generalized seizures, assigning only the most be- 
A
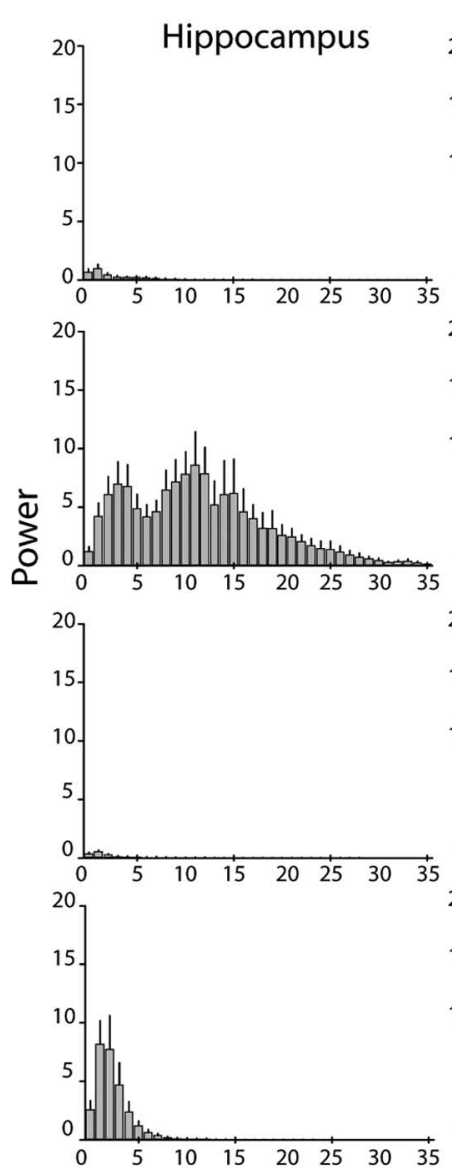

Frequency $(\mathrm{Hz})$
B Secondarily-Generalized Seizures
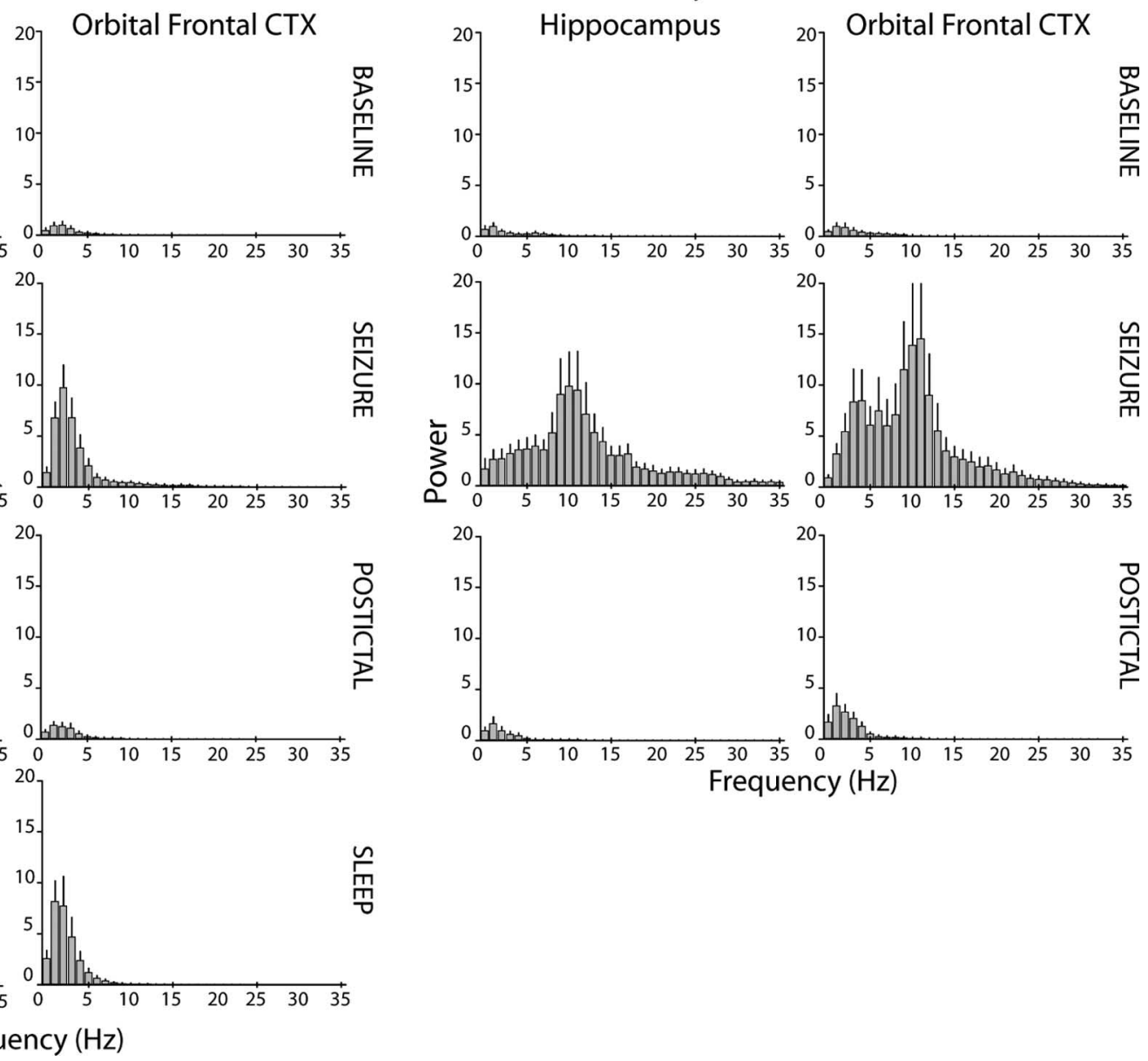

Figure 2. Power spectra showing fast activity in the hippocampus and slow activity in the neocortex during partial seizures. Whereas seizure activity is characterized by dramatic increases in LFP power peaking in the alpha range $(8-13 \mathrm{~Hz})$, ictal neocortical slow activity is associated with large increases in delta $(0.3-4 \mathrm{~Hz})$ power without higher-frequency peaks. All power amplitudes are normalized to the peak amplitude of the baseline power spectrum (peak power at baseline, 1). $A$, Average power spectra of baseline, seizure, postictal, and natural sleep LFP activity corresponding to partial seizures with ictal neocortical slow activity. During partial seizures, hippocampal LFPs increase across the spectrum (second row). The largest peak is seen at $9-12 \mathrm{~Hz}$, corresponding to the most commonly observed spike frequency. Increases in frontal cortical LFP during the seizure peak in the delta range at $1-2 \mathrm{~Hz}$ (second row), corresponding to the observed frequency of ictal neocortical slow oscillations. No high-frequency peaks are observed in frontal power during seizures. The ictal increase in frontal delta activity resembles changes seen during sleep (bottom row). Mean results are shown for $n=12$ seizures in 7 animals. $\boldsymbol{B}$, Average power spectra of baseline, seizure, and postictal activity corresponding to secondarily generalized seizures with frontal propagation of fast seizure activity. During propagating seizures, LFP power in both the hippocampus and frontal cortex (CTX) increases across the spectrum compared with baseline, peaking at 9 - 12 $\mathrm{Hz}$ (second row). Mean results ( + SEM) are shown for $n=37$ seizures in 11 animals.

Table 1. Behavioral rating and EEG classification of spontaneous limbic seizures

\begin{tabular}{llll}
\hline & $\begin{array}{l}\text { Partial seizures with } \\
\text { neocortical slow } \\
\text { activity }(n)\end{array}$ & $\begin{array}{l}\text { Secondarily generalized } \\
\text { seizures }(n)\end{array}$ & $\begin{array}{l}\text { Total } \\
\text { seizures }^{b}(n)\end{array}$ \\
\hline $0 / 1$ & 11 & 0 & 11 \\
2 & 1 & 18 & 19 \\
3 & 0 & 1 & 1 \\
4 & 0 & 6 & 6 \\
5 & 0 & 12 & 12 \\
\hline
\end{tabular}

${ }^{a}$ Behavioral seizure rating assessed using the methods of Racine (1972), with class 0 and 1 seizures combined into one category.

${ }^{b} n=49$ seizures from 14 pilocarpine-treated animals.

haviorally severe seizures (class 4-5) to the "convulsive" seizure group and all milder seizures (class $0-3$ ) to the "nonmotor" seizure group, also revealed a significant relationship $\left(\chi^{2}=9.2 ; p<\right.$ 0.01 ). This finding suggests that, whereas electrographically generalized seizures are associated with varying degrees of convulsive activity, electrographically partial seizures are characterized by milder staring spells and automatisms.
Electrically stimulated partial limbic seizures are associated with ictal neocortical slow activity

We next sought an acute animal model of ictal neocortical slow activity, which would allow both greater control over seizures and also the ability to invasively measure associated changes in neuronal activity and hemodynamic responses. It was found that in the lightly anesthetized rat, electrically stimulating the hippocampus using a low-intensity stimulus consistently produced partial limbic seizures associated with ictal neocortical slow waves, whereas a higher intensity stimulus generated secondarily generalized seizures with cortical propagation of seizure activity.

During 17 partial seizures recorded in 12 rats, hippocampal activity was characterized by fast $9-12 \mathrm{~Hz}$ polyspike activity in LFPs and large population spikes in MUA ictally, whereas LFPs in the orbitofrontal cortex showed large-amplitude $1-2 \mathrm{~Hz}$ slow oscillations (Fig. $3 A$ ) similar to those seen during spontaneous seizures. Although postictal depression of neuronal firing was seen in the hippocampus, frontal slow oscillations continued postictally. Ictal and postictal MUA recordings in the frontal cor- 

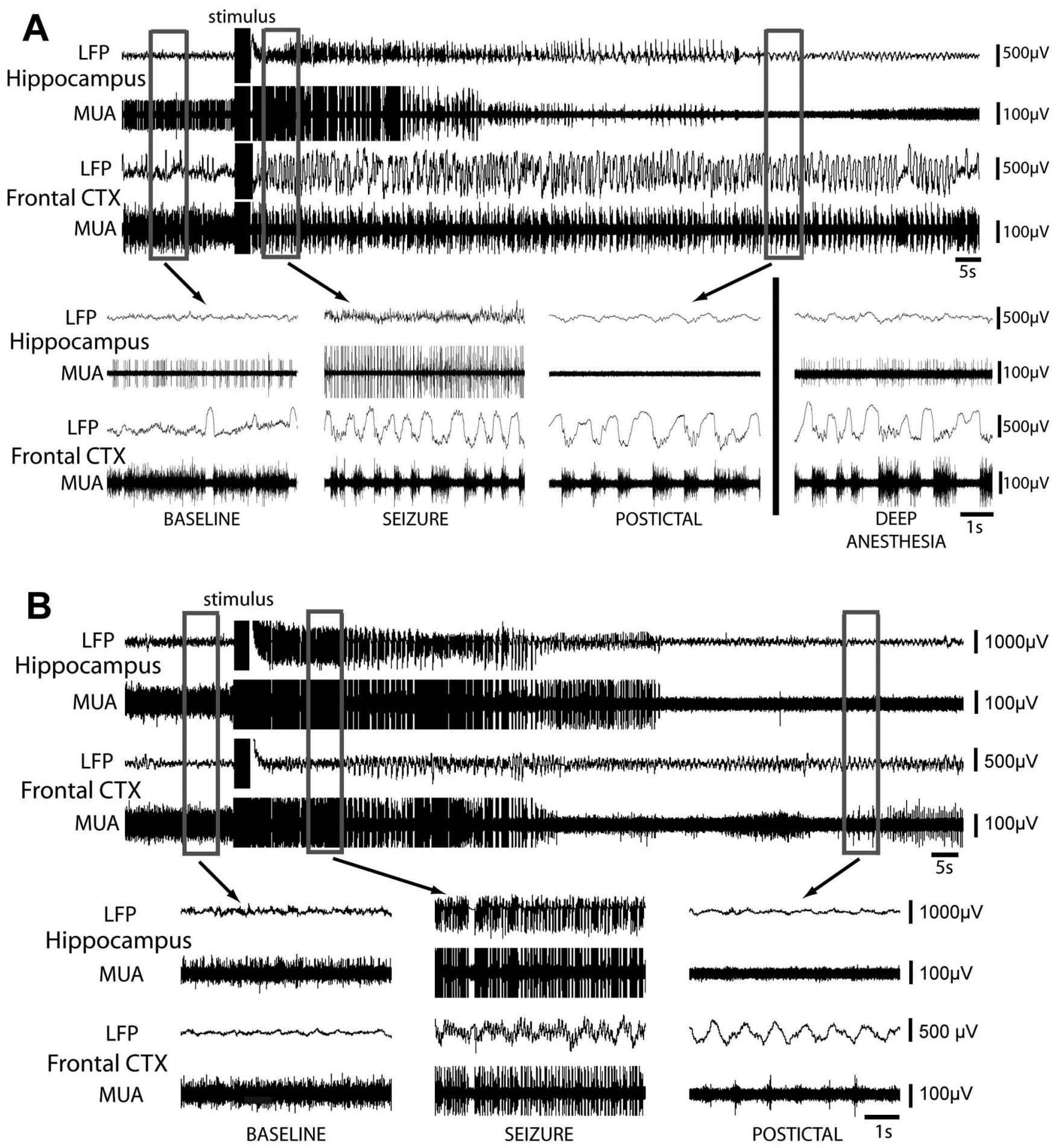

Figure 3. Electrically stimulated partial limbic seizures produce ictal neocortical slow waves in the orbitofrontal cortex (CTX), whereas secondarily generalized seizures show fast polyspike activity. A, Example of LFP and MUA recordings during a low-current stimulated partial seizure in a lightly anesthetized rat. During baseline, recordings show a stable theta rhythm in hippocampal LFP and low-voltage beta activity with occasional slow waves in the orbitofrontal cortex (see also inset). MUA recordings reveal relatively stable neuronal firing in both areas. During the seizure, hippocampal LFP recordings show 9-10 Hz fast polyspike activity ictally associated with population spikes in MUA recordings. Population spikes are often up to 10 times larger in amplitude than individual baseline units and are thus shown truncated here. In the orbitofrontal cortex, 1-2 Hz large-amplitude slow waves are seen in LFP recordings, associated with up and down states of neuronal firing in MUA recordings. No fast polyspike activity is present in the frontal cortex LFPs. After the seizure, hippocampal activity is depressed whereas frontal slow oscillations persist postictally. Recordings from the same rat under deep anesthesia at a different time are also shown (bottom right), during which slow activity is present in the frontal cortex. $\boldsymbol{B}$, Example of LFP and MUA recordings during a high-current stimulated secondarily generalized seizure. During the seizure, $10-11 \mathrm{~Hz}$ fast polyspike seizure is observed in both brain regions, associated with population spikes in MUA recordings in both locations. As in $\boldsymbol{A}$, population spikes are shown truncated here. Up and down states of neuronal firing are not seen in the frontal recording ictally. Postictally, large-amplitude slow activity is seen in the orbitofrontal cortex. LFP recordings are filtered $0.1-100 \mathrm{~Hz}$ and MUA recordings are filtered $400 \mathrm{~Hz}$ to $20 \mathrm{kHz}$. 
tex revealed that slow waves were associated with up and down states of neuronal firing (Fig. $3 A$, frontal MUA tracings), resembling the up and down states reported previously in other forms of cortical slow oscillations (Steriade et al., 1993; Cowan and Wilson, 1994; Haider et al., 2006). As in these previous reports, we observed that each positive slow wave corresponded to a distinct down state of reduced neuronal firing. We also recorded a similar slow rhythm in the frontal cortex without seizure activity when rats were placed under deep ketamine/xylazine anesthesia (Fig. $3 A$, bottom right).

In addition to partial seizures, 15 recordings of secondarily generalizing seizures were collected in eight rats. During generalized seizures, fast polyspike seizure activity in both hippocampus and frontal cortex was associated with population spikes in MUA recordings (Fig. $3 B$ ). No down states of neuronal firing were seen in the frontal cortex ictally. Postictally, hippocampal recordings revealed suppression of MUA and emergence of low-amplitude LFP slow activity, whereas large-amplitude slow LFP oscillations were seen in the orbitofrontal cortex (Fig. $3 B$, bottom right).

Summary power spectra of electrically stimulated seizures in lightly anesthetized animals (supplemental Fig. 3, available at www.jneurosci.org as supplemental material) closely resembled power spectra of spontaneous partial and generalized seizures recorded in pilocarpine-treated rats (Fig. 2). Furthermore, elevated delta-range frontal power during acutely stimulated partial seizures resembled similar changes in delta power observed during deep anesthesia (supplemental Fig. 3A, available at www. jneurosci.org as supplemental material) and during slow-wave sleep (Fig. $2 A$ ). These observations during both spontaneous and stimulated partial seizures suggest that ictal neocortical slow waves represent a distinct physiological state, differing from the high-frequency activity typically seen during seizure propagation.

\section{Ictal neocortical slow activity results in decreased BOLD and CBV}

To further characterize and map the effects of limbic seizures on distal brain networks, we performed four types of fMRI experiments in lightly anesthetized rats. We measured BOLD signal changes throughout the brain during partial $(n=11$ experiments) and secondarily generalized $(n=11)$ electrically stimulated seizures, and we also measured CBV signal changes during both seizure types ( $n=10$ partial, 17 generalized). As in acute bench experiments, lower stimulus intensities were used to generate partial seizures and higher currents were administered to induce generalized seizures. Whereas raw EEG and hippocampal LFP signals during fMRI recording revealed prominent MRI artifacts during each image acquisition, applying a low-pass filter to these signals dramatically reduced artifacts to allow clear identification of seizure duration and hippocampal seizure activity (supplemental Fig. 1, available at www.jneurosci.org as supplemental material).

The mean duration ( \pm SEM) of electrically stimulated partial seizures was $60 \pm 5 \mathrm{~s}$. These events were associated with BOLD signal increases in the bilateral hippocampus, as well as the thalamus and septal nuclei (Fig. $4 A$ ). In contrast, prominent BOLD decreases were seen in distal cortical regions such as the orbitofrontal, anterior cingulate, and retrosplenial/posterior cingulate cortices (Fig. 4A). Secondarily generalized seizures, which had a mean duration of $59 \pm 5 \mathrm{~s}( \pm \mathrm{SEM})$, were also characterized by BOLD activations in the hippocampus, thalamus, and septal nuclei, although changes in these regions appeared more intense than during partial seizures (Fig. $4 B$ ). Widespread cortical in- creases in signal were also observed ictally, especially in the orbitofrontal, cingulate, and primary somatosensory cortices, with relatively few regions of decreases (Fig. $4 B$ ). The BOLD imaging results again suggest that partial limbic seizures may have remote network effects leading to deactivation of several cortical regions including the orbitofrontal cortex. Generalized seizures, however, lead directly to abnormally increased activity of these same cortical regions.

Because BOLD signals are only indirectly related to neuronal activity (Hyder et al., 2002; Englot and Blumenfeld, 2008) and do not always accurately reflect changes in neuronal activity or vascular responses (Schridde et al., 2008), we also measured changes in the flow-related CBV in the same animals after injection of exogenous paramagnetic contrast. Changes in CBV during hippocampal-stimulated seizures closely mirrored BOLD signal alterations. Partial seizures showed increased CBV in the hippocampus, thalamus, and septal nuclei, along with decreased $\mathrm{CBV}$ in the orbitofrontal, cingulate, and retrosplenial cortices (Fig. 5A). During generalized seizures, there were dramatic CBV increases in the hippocampus, thalamus, and septal nuclei, as well as in several cortical regions including the orbitofrontal cortex (Fig. 5B). These findings suggest that changes in BOLD signals during limbic seizures are accompanied by concomitant hemodynamic changes in the same direction. However, to investigate this more fully, we next analyzed the time courses of neuroimaging signals, so these could be compared with direct physiological recordings of neuronal activity.

To characterize the time course of BOLD and CBV signal changes during limbic seizures, we aligned all runs to time of stimulus and plotted mean signals ( \pm SEM) from six ROIs (supplemental Fig. 2, available at www.jneurosci.org as supplemental material) as percentage change from prestimulus baseline (Fig. 6). During partial seizures, mean BOLD and CBV signal elevations in the hippocampus, thalamus, and septal nuclei all peaked during mid to late seizure and then declined toward baseline postictally (Fig. 6A, left column). Simultaneously, BOLD and CBV signals decreased significantly in the orbitofrontal, cingulate, and retrosplenial cortex during the seizures, and slowly returned toward baseline after the seizures (Fig. $6 \mathrm{~A}$, right column). In secondarily generalized seizures, the magnitudes of BOLD and CBV increases in the hippocampus, thalamus, and septal nuclei were generally larger than during partial seizures (Fig. $6 \mathrm{~B}$, left column). Interrogation of all three cortical ROIs revealed considerable increases in BOLD and CBV during seizures (Fig. 6 B, right column), which differed significantly from neocortical decreases observed during partial seizures $(p<0.001$ for each comparison, unpaired $t$ tests). The mean percentage changes in BOLD and CBV signals during the first $45 \mathrm{~s}$ of seizure versus $45 \mathrm{~s}$ baseline were statistically significant during both partial and generalized seizures, as summarized in Table 2 (first two columns). The time courses of these neuroimaging signals were next compared with direct physiological recordings.

\section{Neuronal activity and CBF decrease in the neocortex during partial seizures}

The above findings suggest that neocortical slow waves during partial limbic seizures differ from propagated seizure activity in terms of power spectral characteristics and neuroimaging signals. However, how are altered neuronal firing patterns during this state of slow oscillations related to decreased neocortical function? As discussed above, ictal neocortical slow waves are accompanied by alternating increases and decreases in neuronal firing, 


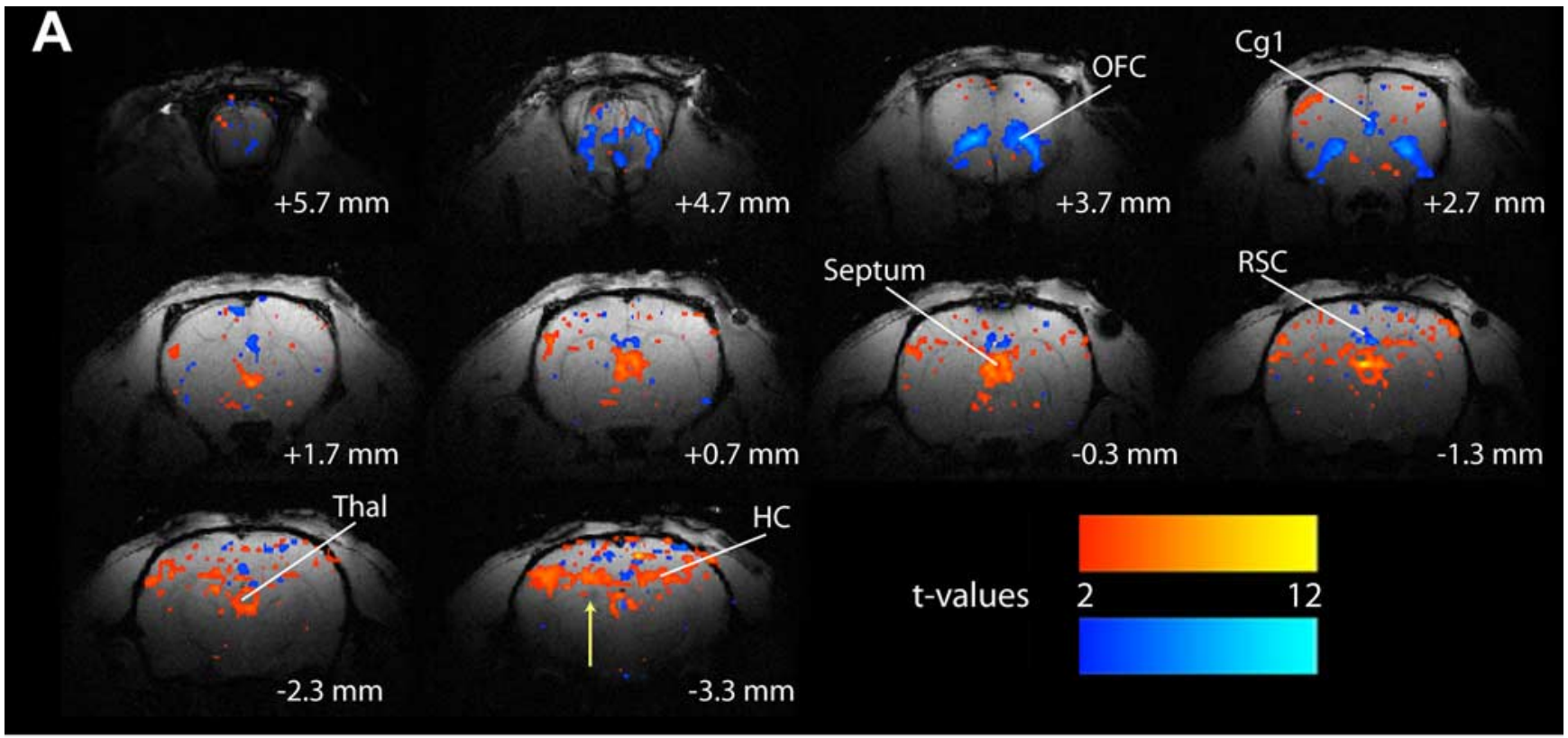

B

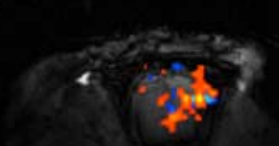

$+5.7 \mathrm{~mm}$

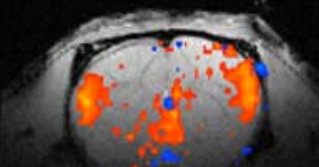

$+1.7 \mathrm{~mm}$

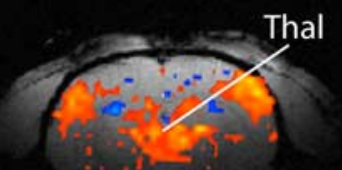

$-2.3 \mathrm{~mm}$

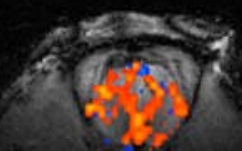

$+4.7 \mathrm{~mm}$

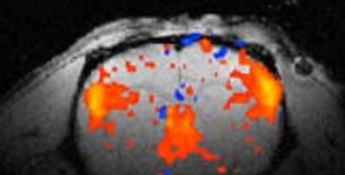

$+0.7 \mathrm{~mm}$

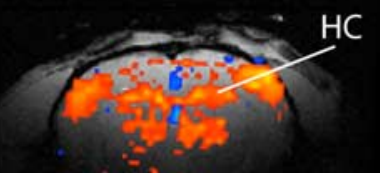

$-3.3 \mathrm{~mm}$

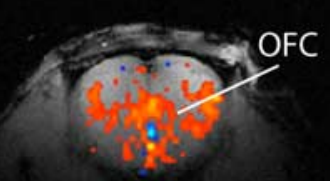

$+3.7 \mathrm{~mm}$

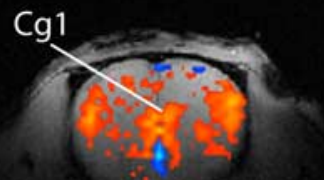

$+2.7 \mathrm{~mm}$
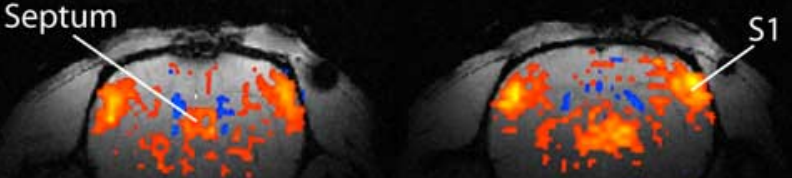

$-0.3 \mathrm{~mm}$

$-1.3 \mathrm{~mm}$

Figure 4. Example of $B O L D$ increases and decreases during electrically stimulated partial and secondarily generalized limbic seizures. $A$, During partial limbic seizures, BOLD fMRI signal increases are observed in the hippocampus, thalamus, and septal nuclei. Prominent BOLD decreases are seen in the orbitofrontal, anterior cingulate, and retrosplenial/posterior cingulate cortices. The arrow signifies the hippocampal electrode artifact. $\boldsymbol{B}$, During secondarily generalized limbic seizures, BOLD signal increases are observed in the hippocampus, thalamus, and septal region, and appear more intense than increases seen in those regions during partial seizures $(\boldsymbol{A})$. In addition, BOLD increases are observed in widespread cortical regions, including the orbitofrontal, cingulate, and primary somatosensory cortices. Few regions of BOLD decreases are observed. $t$-Maps are shown for the first $30 \mathrm{~s}$ of seizure activity ( 10 consecutive fMRI images acquired every $3 \mathrm{~s}$ ) versus $30 \mathrm{~s}$ baseline and are superimposed on high-resolution anatomical images. Slices are shown from anterior to posterior, with approximate coordinates relative to bregma (Paxinos and Watson, 1998). Color bars indicate $t$-values for increases (warm colors) and decreases (cold colors). The display threshold is $t=2$. Cg1, Anterior cingulate cortex; $\mathrm{HC}$, hippocampus; OFC, orbitofrontal cortex; $\mathrm{RSC}$, retrosplenial/ posterior cingulate cortex; septum, Septal nuclei; S1, primary somatosensory cortex; Thal, thalamus.

resembling the up and down states seen in sleep and deep anesthesia (Fig. 3A, MUA in bottom trace). To further investigate the effects of this altered state on cortical physiology, we generated time courses of mean percentage signal change $( \pm$ SEM) of direct electrophysiological measurements of neuronal activity (LFPs and MUA) and CBF measured using LDF in both the hippocampus and frontal cortex (Fig. 7).

During electrically stimulated partial seizures in lightly anesthetized rats, dramatic increases in hippocampal LFPs, MUA, and
CBF were observed ictally during fast polyspike seizure activity, with a return toward baseline postictally (Fig. $7 A$, left column). In the orbitofrontal cortex, however, although ictal neocortical slow waves during partial seizures were associated with increased LFP amplitude, we observed consistent decreases in MUA and CBF ictally (Fig. 7A, right column). These signals remained depressed postictally, and gradually returned toward baseline over several minutes. In secondarily generalized seizures, LFPs, MUA, and $\mathrm{CBF}$ all increased in both the hippocampus and frontal cortex 

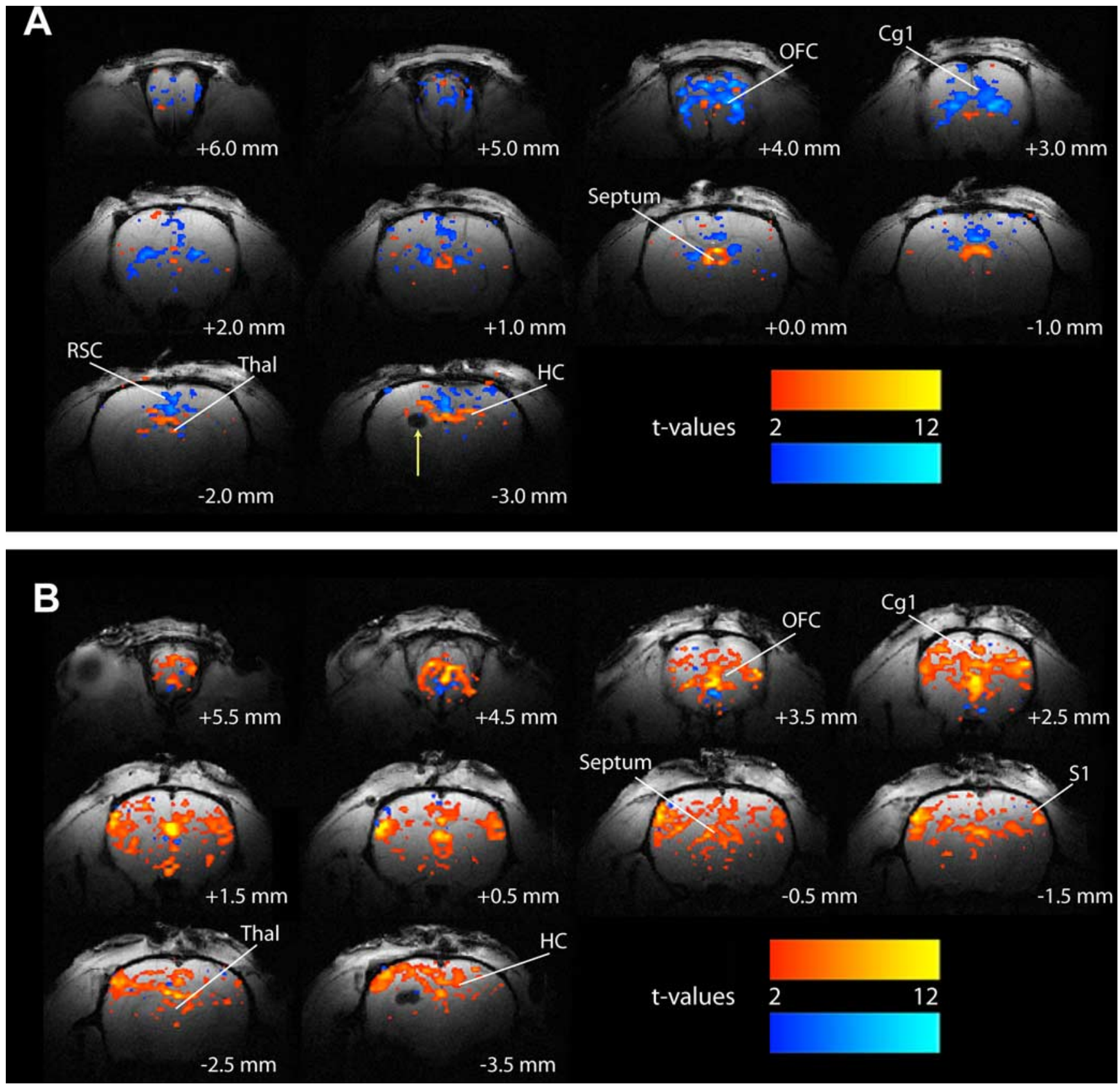

Figure 5. Example of CBV increases and decreases measured using fMRI during electrically stimulated partial and secondarily generalized limbic seizures. $A$, During partial limbic seizures, CBV signal increases are observed in the hippocampus, thalamus, and septal nuclei, whereas decreases are seen in the orbitofrontal, anterior cingulate, and retrosplenial cortices. The arrow signifies hippocampal electrode artifact. $\boldsymbol{B}$, During secondarily generalized limbic seizures, CBV increases are observed in the hippocampus, thalamus, and septal region, as well as in widespread cortical regions, including the orbitofrontal, cingulate, and primary somatosensory cortices. Few CBV decreases are seen. CBV changes during both seizure types closely mirror BOLD signal changes (Fig. 4). $t$-Maps are shown for the first $30 \mathrm{~s}$ of seizure activity (10 consecutive fMRl images acquired every $3 \mathrm{~s}$ ) versus $30 \mathrm{~s}$ baseline, and are superimposed on high-resolution anatomical images. Slices are shown from anterior to posterior, with approximate coordinates relative to bregma (Paxinos and Watson, 1998). Color bars indicate $t$ values for increases (warm colors) and decreases (cold colors). Note that although intravenous paramagnetic contrast results in decreased signal with increased CBV, color-labeled changes have been inverted for clarity so that warm colors indicate CBV increases. The display threshold is $t=2$. Cg1, Anterior cingulate cortex; HC, hippocampus; OFC, orbitofrontal cortex; RSC, retrosplenial/posterior cingulate cortex; Septum, septal nuclei; S1, primary somatosensory cortex; Thal, thalamus.

during the ictal period (Fig. $7 B$ ). The changes in frontal cortical MUA and CBF thus differed markedly during partial and secondarily generalized seizures $(p<0.001$ for each comparison, unpaired two-tailed $t$ tests). All signal changes in frontal cortex and hippocampus during both seizure types were statistically significant, as summarized in Table 2 (third, fourth, and fifth columns).
Ictal neocortical slow activity: neuronal activity and oxidative metabolism resemble sleep, not seizures

$\mathrm{CMRO}_{2}$ reflects cerebral metabolism, and it is the most direct neuroimaging measurement of neuronal activity (Kida and Hyder, 2006). Using Equation 1 (Materials and Methods), we combined BOLD and CBV signal data from imaging experiments with LDF data from bench experiments to estimate mean per- 
A
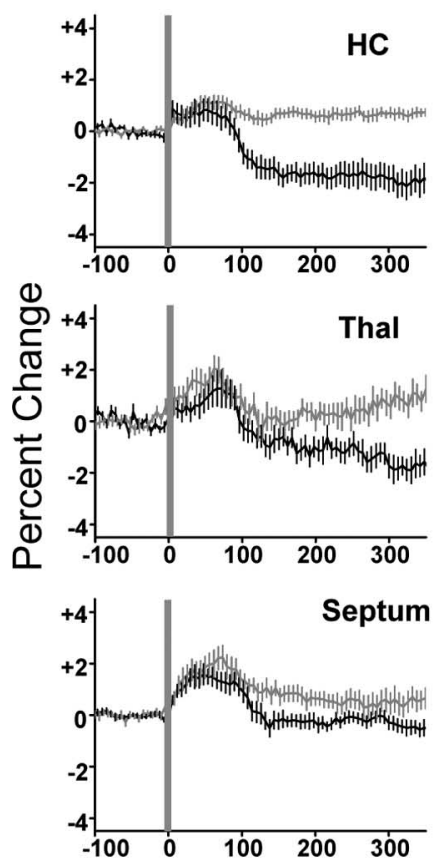

Partial Seizures
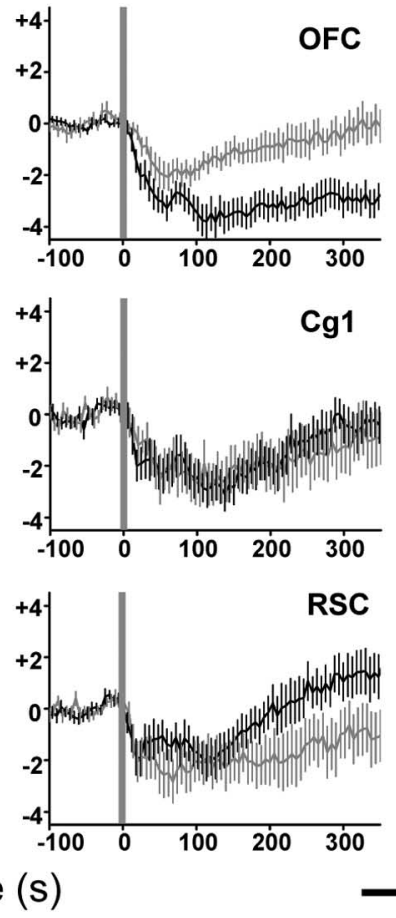

B
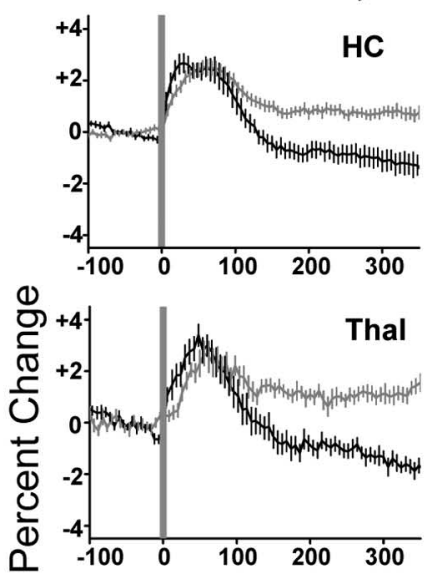

Thal

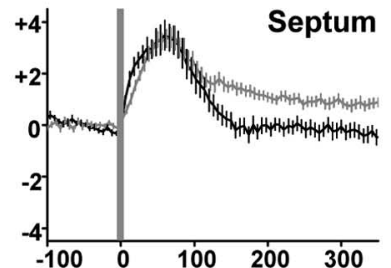

Time (s)

Figure 6. Mean time courses of $B O L D$ and CBV signal changes during partial and secondarily generalized seizures. $A, B O L D$ and CBV changes during partial seizures. BOLD signals increase in the hippocampus, thalamus, and septal nuclei during partial seizures, with similar changes in CBV. In contrast, BOLD signals and CBV decrease during seizures in the orbitofrontal, cingulate, and retrosplenial cortices. BOLD data are from 11 seizures in 8 animals and CBV data are from 10 seizures in 5 animals. B, BOLD and CBV changes during secondarily generalized seizures. BOLD signals increase in the hippocampus, thalamus, and septal nuclei during secondarily generalized seizures, with larger changes observed than during partial seizures $(\boldsymbol{A})$. In addition, BOLD signals increase in the orbitofrontal, cingulate, and retrosplenial cortices ictally. Comparable changes are observed in CBV in all regions. BOLD data are from 11 seizures in 6 animals and CBV data are from 17 seizures in 8 animals. For $A$ and $B$, all average signal changes during the first $45 \mathrm{~s}$ of seizure versus 45 s baseline were statistically significant (Table 2). Vertical gray lines represent the synchronized beginning of all seizures in each data set. All graphs show mean ( \pm SEM) percentage change in signal from a $30 \mathrm{~s}$ baseline, binned at $6 \mathrm{~s}$ intervals. $\mathrm{Cg} 1$, Anterior cingulate cortex; HC, hippocampus; $0 \mathrm{FC}$, orbitofrontal cortex; RSC, retrosplenial/posterior cingulate cortex; Septum, septal nuclei; Thal, thalamus.

centage change of $\mathrm{CMRO}_{2}( \pm \mathrm{SEM})$ in the hippocampal and orbitofrontal cortex during both partial and generalized seizures (Fig. $7 A, B$, bottom rows). During partial seizures, ictal and postictal neocortical slow activity were associated with decreased $\mathrm{CMRO}_{2}$ in the frontal cortex, closely mirroring changes MUA and $\mathrm{CBF}$ (Fig. 7A, bottom right). However, fast polyspike seizure activity, which was present in the hippocampus during partial seizures (Fig. 7A, bottom left) and in both the hippocampus and frontal cortex during generalized seizures (Fig. $7 B$, bottom row), was consistently associated with elevated $\mathrm{CMRO}_{2}$ ictally. $\mathrm{CMRO}_{2}$ changes in the hippocampus and frontal cortex were statistically significant for both seizures types (Table 2, right column). Thus, unlike $\mathrm{CMRO}_{2}$ increases normally associated with partial or generalized seizure activity in hippocampus or cortex, we observed that ictal neocortical slow waves were associated with $\mathrm{CMRO}_{2}$ decreases.

As discussed above, the frequency composition of ictal neocortical slow activity more closely resembles sleep (Figs. $1 A, 2 A$ ) or deep anesthesia (Fig. $3 A$, supplemental Fig. $3 A$, available at www.jneurosci.org as supplemental material) than seizures, and is also similar to slow activity seen in the postictal period. On a more fundamental level, it was observed that changes in frontal LFPs, MUA, and CBF during ictal neocortical slow activity closely resembled similar signal changes seen during the postictal period and deep anesthesia (Fig. 8).

\section{Discussion}

Partial limbic seizures are associated with slow oscillations and decreased neuronal activity in the neocortex

Limbic seizures in TLE are typically classified as partial or secondarily generalized. One poorly understood problem, however, is how nongeneralizing partial hippocampal seizures affect distant neocortical networks. This may be a particularly important clinical question, as previous investigations have found neocortical deficits such as gray matter atrophy (Bonilha et al., 2006) and interictal hypometabolism (Diehl et al., 2003; Nelissen et al., 2006) in TLE patients, which may be related to cognitive impairments and neuropsychological sequelae often suffered by these individuals (Helmstaedter and Kockelmann, 2006; Laurent and Arzimanoglou, 2006). In addition, ictal and postictal neocortical slow activity, depressed blood flow, and metabolism may be related to impaired consciousness commonly seen during and after temporal lobe seizures (Blumenfeld et al., 2004a,b)

In the present study, we performed a multimodal investigation of partial and secondarily generalized seizures in rats, using chronic video/EEG and acute electrophysiology recordings, as well as indirect (fMRI) and direct (LDF) assessments of cerebral vascular responses. In awake-behaving rats, seizures which propagated to the frontal cortex were associated with behavioral convulsions. However, partial seizures with frontal slow activity were characterized by milder behavioral manifestations, such as star- 
Table 2. Mean percentage change of neuroimaging, electrophysiology, and LDF signals during seizures

\begin{tabular}{|c|c|c|c|c|c|c|}
\hline \multirow[t]{2}{*}{ Region of interest } & \multicolumn{6}{|c|}{ Percentage signal change during seizure \pm SEM $(n)$ and significance level $p$} \\
\hline & BOLD & CBV & LFPs & MUA & CBF & $\mathrm{CMRO}_{2}$ \\
\hline \multicolumn{7}{|l|}{ Partial seizures versus baseline } \\
\hline Hippocampus & $\begin{array}{l}0.85 \pm 0.40(11) \\
p=0.04\end{array}$ & $\begin{array}{l}0.82 \pm 0.20(10) \\
p<0.01\end{array}$ & $\begin{array}{c}291 \pm 88(17) \\
p<0.01\end{array}$ & $\begin{aligned} 729 & \pm 254(17) \\
p & =0.01\end{aligned}$ & $\begin{array}{c}65.6 \pm 9.0(15) \\
p<0.001\end{array}$ & $\begin{array}{c}55.4 \pm 12.0(10) \\
p<0.001\end{array}$ \\
\hline Thalamus & $\begin{array}{l}0.73 \pm 0.33(11) \\
p<0.05\end{array}$ & $\begin{array}{c}1.13 \pm 0.44(10) \\
p=0.02\end{array}$ & & & & \\
\hline Septal nuclei & $\begin{array}{l}1.18 \pm 0.27(11) \\
p<0.001\end{array}$ & $\begin{array}{l}1.37 \pm 0.35(10) \\
p<0.01\end{array}$ & & & & \\
\hline Orbitofrontal cortex & $\begin{array}{l}-1.94 \pm 0.51(11) \\
p<0.01\end{array}$ & $\begin{array}{l}-1.23 \pm 0.45(10) \\
p=0.02\end{array}$ & $\begin{array}{c}119 \pm 14(17) \\
p<0.001\end{array}$ & $\begin{array}{l}-23.4 \pm 2.3(17) \\
p<0.001\end{array}$ & $\begin{array}{l}-14.5 \pm 2.1(15) \\
p<0.001\end{array}$ & $\begin{array}{l}-9.64 \pm 3.0(10) \\
p<0.001\end{array}$ \\
\hline Anterior cingulate cortex & $\begin{array}{l}-1.66 \pm 0.65(11) \\
p=0.01\end{array}$ & $\begin{array}{l}-1.65 \pm 0.62(10) \\
p=0.02\end{array}$ & & & & \\
\hline $\begin{array}{l}\text { Retrosplenial / posterior cingulate } \\
\text { cortex }\end{array}$ & $\begin{array}{l}-1.69 \pm 0.56(11) \\
p=0.01\end{array}$ & $\begin{array}{l}-1.96 \pm 0.72(10) \\
\quad p=0.02\end{array}$ & & & & \\
\hline \multicolumn{7}{|c|}{ Secondarily generalizing seizures versus baseline } \\
\hline Hippocampus & $\begin{array}{c}2.49 \pm 0.29(11) \\
p<0.001\end{array}$ & $\begin{array}{c}1.67 \pm 0.32(17) \\
p<0.001\end{array}$ & $\begin{array}{c}211 \pm 14(17) \\
p<0.001\end{array}$ & $\begin{array}{c}971 \pm 190(17) \\
p<0.001\end{array}$ & $\begin{array}{c}71.1 \pm 12(17) \\
p<0.001\end{array}$ & $\begin{array}{c}60.0 \pm 11(11) \\
p<0.001\end{array}$ \\
\hline Thalamus & $\begin{array}{c}2.45 \pm 0.35(11) \\
p<0.001\end{array}$ & $\begin{array}{c}1.00 \pm 0.33(17) \\
p<0.001\end{array}$ & & & & \\
\hline Septal nuclei & $\begin{array}{c}2.56 \pm 0.26(11) \\
p<0.001\end{array}$ & $\begin{array}{c}1.77 \pm 0.24(17) \\
p<0.001\end{array}$ & & & & \\
\hline Orbitofrontal cortex & $\begin{array}{c}2.77 \pm 0.38(11) \\
p<0.001\end{array}$ & $\begin{array}{c}1.47 \pm 0.16(17) \\
p<0.001\end{array}$ & $\begin{array}{c}130 \pm 30(17) \\
p<0.001\end{array}$ & $\begin{array}{c}36.0 \pm 12(17) \\
p<0.01\end{array}$ & $\begin{array}{c}45.4 \pm 9.0(17) \\
p<0.001\end{array}$ & $\begin{array}{c}41.1 \pm 8.2(11) \\
p<0.001\end{array}$ \\
\hline Anterior cingulate cortex & $\begin{array}{c}2.38 \pm 0.62(11) \\
p<0001\end{array}$ & $\begin{array}{c}2.15 \pm 0.34(17) \\
p<0.001\end{array}$ & & & & \\
\hline $\begin{array}{l}\text { Retrosplenial /posterior cingulate } \\
\text { cortex }\end{array}$ & $\begin{array}{l}2.17 \pm 0.27(11) \\
p<0.001\end{array}$ & $\begin{array}{c}1.71 \pm 0.47(17) \\
p<0.001\end{array}$ & & & & \\
\hline
\end{tabular}

All significance levels $(p)$ were measured using a two-tailed $t$ test of the last $45 \mathrm{~s}$ of baseline signal before stimulus versus the first $45 \mathrm{~s}$ of seizure activity. $n$ is the number of seizures in which measurements were performed for each region.

ing and facial automatisms. We also observed during stimulated seizures in lightly anesthetized animals that fast polyspike seizure activity was associated with significant elevations in electrophysiological responses (LFPs, MUA), hemodynamic measurements (BOLD, CBV , CBF), and cerebral metabolism $\left(\mathrm{CMRO}_{2}\right)$. In contrast, ictal neocortical slow activity was characterized by significant decreases in neuronal firing (MUA), hemodynamic measurements (BOLD, CBV, CBF), and $\mathrm{CMRO}_{2}$. These results suggest that ictal neocortical slow oscillations during partial nonconvulsive seizures are associated with an overall decrease in neuronal activity and metabolism in the frontal cortex.

Similar slow LFP deflections have been observed during spontaneous slow oscillations, as seen during slow-wave sleep and deep anesthesia (Steriade et al., 1993; Haider et al., 2006). These large-amplitude, positive LFP deflections are invariably associated with down states that are characterized by reduced cortical excitability and neuronal silence (Bazhenov et al., 2002; McCormick, 2005) that dominates most of the oscillation cycle. Thus, despite large fluctuating LFP signals during ictal slow oscillations in the frontal cortex, there is an overall decrease in neuronal firing, blood flow, and metabolism.

\section{Ictal slow waves represent a depressed neocortical state, not propagation of seizure activity}

Although evidence for ictal neocortical slow activity in complex partial temporal lobe seizures has been uncovered in several human intracranial EEG studies (Lieb et al., 1991; Franaszczuk et al., 1994; Mayanagi et al., 1996; Eisenschenk et al., 2001), it has typically been interpreted as propagation of seizure activity. However, no direct measurements of neuronal activity during ictal neocortical slow activity have been reported previously. Although a few animal studies have reported regions of depressed neuronal activity related to seizures, these have focused on either 'surround inhibition' of small areas immediately surrounding an epileptic focus (Prince and Wilder, 1967; Schwartz and Bonhoeffer, 2001; Bahar et al., 2006), or hypometabolism after prolonged seizure activity (Collins, 1978; Handforth and Ackermann, 1988; VanLandingham and Lothman, 1991; Bruehl et al., 1995; Redecker et al., 1997). These studies did not investigate long-range cortical depression during partial limbic seizures. Furthermore, whereas initial slow waves (ISWs) have been described at a seizure focus before seizure onset (Bragin et al., 2007), ictal slow activity during partial limbic seizures occurs in distal cortical structures during and after the seizure.

Notably, we recently observed BOLD decreases in the rat hippocampus during primary generalized tonic-clonic seizures caused by acute bicuculline injection (Schridde et al., 2008). However, these hippocampal BOLD decreases were associated with dramatic increases in neuronal activity, CBF, CBV, and $\mathrm{CMRO}_{2}$, opposite to the changes associated with BOLD deactivations that we observed during ictal neocortical slow activity. In bicuculline-induced tonic-clonic seizures, it appears that intense hippocampal seizure activity causes a dramatic elevation in cerebral metabolism that overwhelms reflexive hemodynamic responses, resulting in an "uncoupling" of the BOLD signal from neuronal activity and metabolism (Schridde et al., 2008). It is therefore important to recognize the difference between BOLD decreases, which result from paradoxical signal uncoupling, and BOLD deactivations in the present study, in which fMRI decreases remain closely coupled to depressed neuronal activity, hemodynamic responses, and cerebral metabolism.

Our results suggest that neocortical slow activity during complex partial seizures represents an abnormal state of depressed cortical function that is distinct from excitatory seizure activity. 
A

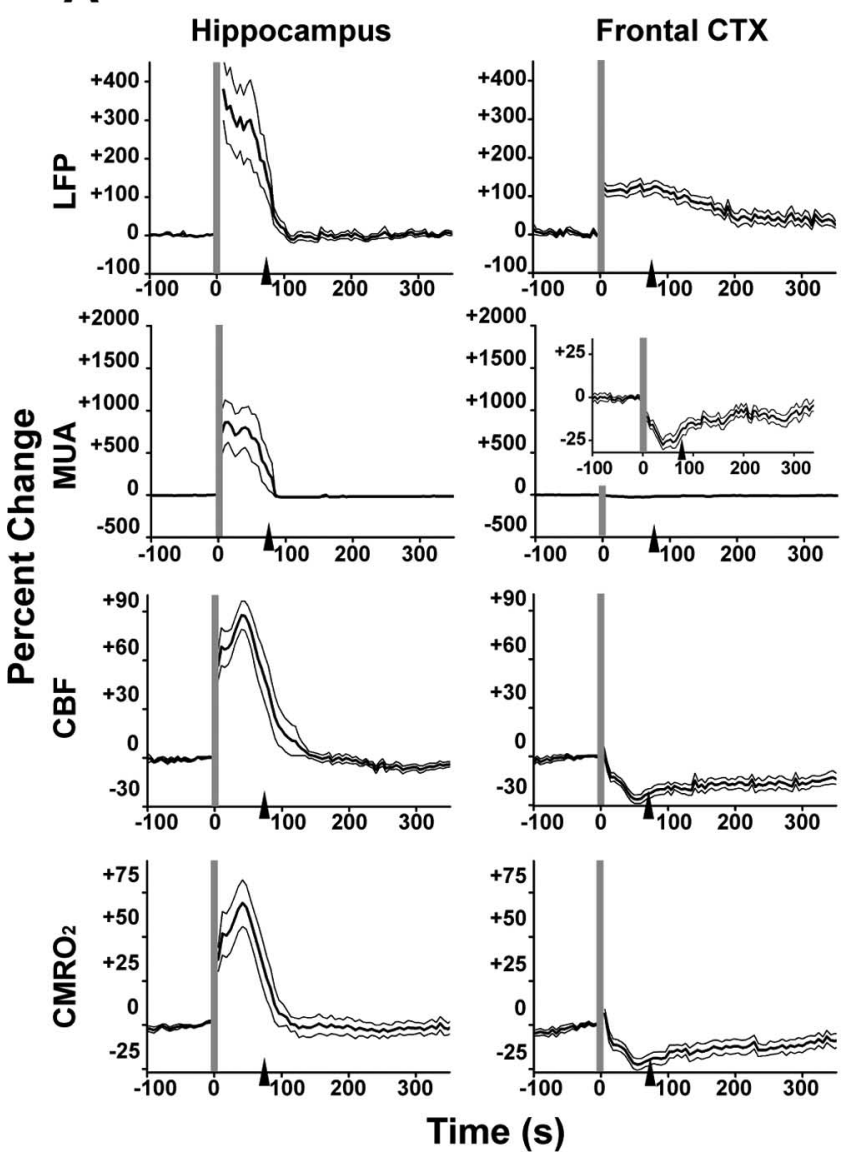

B Secondarily-Generalized Seizures Hippocampus

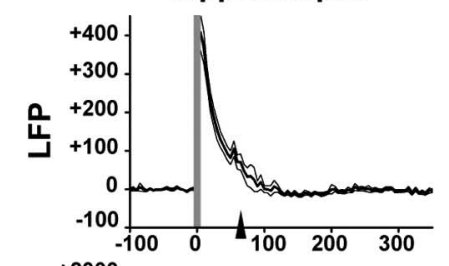
Frontal CTX
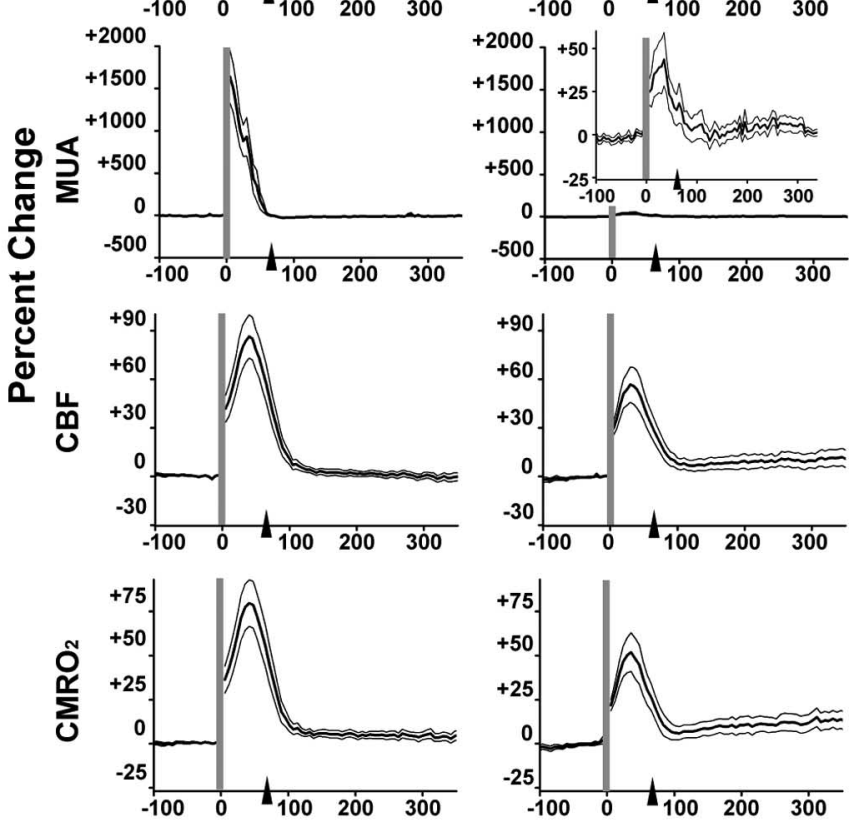

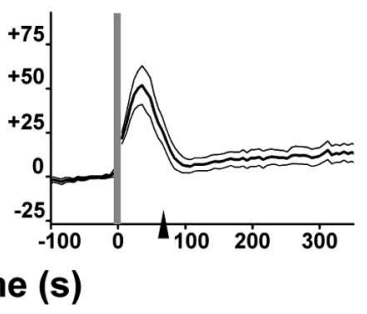

Figure 7. Neuronal activity, CBF, and cerebral metabolism differ markedly during ictal neocortical slow activity vs fast seizure activity. $\boldsymbol{A}$, Mean electrophysiology (MUA and LFPs), CBF, and CMRO, signal changes during partial limbic seizures. In the hippocampus, all parameters increase during seizure, and return toward baseline relatively rapidly postictally. In the frontal cortex (CTX), although there is an increase in LFPs ictally, reflecting large-amplitude slow oscillations, MUA, CBF, and CMRO 2 all decrease during the seizure, with slow recovery. LFP and MUA data are from 17 seizures in 12 animals, $C B F$ data are from 15 seizures in 9 animals, and $\mathrm{CMRO}_{2}$ data are from 10 seizures in 6 animals. $\boldsymbol{B}$, Mean electrophysiology, $C B F$, and CMRO ${ }_{2}$ signal changes during secondarily generalized limbic seizures. In both the hippocampus and frontal cortex, LFPs, MUA, CBF, and CMRO all increase during seizures. LFPs, MUA, and CBF data are from 17 seizures in 8 animals and CMRO data are from 11 seizures in 6 animals. Vertical gray lines represent synchronized beginning and arrowheads signify mean end of all seizures in each data set. All graphs show the mean ( \pm SEM) percentage change from a 30 s baseline, binned at 5 s intervals. Insets in MUA recordings show enlarged graphs to highlight signal changes.

This conclusion is supported by the dramatic differences we observed between the elevated multiunit, hemodynamic, and metabolic responses associated with fast polyspike activity in the hippocampus and the corresponding decreases associated with large-amplitude slow waves in the frontal cortex during partial limbic seizures. Furthermore, whereas cortical seizure propagation is associated with elevated neuronal activity and cerebral metabolism, resembling hippocampal seizure activity, ictal neocortical slow activity more closely resembles slow oscillations seen in humans during various states of depressed brain function, such as slow-wave sleep (Achermann and Borbely, 1997), encephalopathy (Plum and Posner, 1980; Ebersole and Pedley, 2003), and surgical anesthesia (Clark and Rosner, 1973; Burchiel et al., 1977). Interestingly, we observed that neocortical slow waves and depressed neuronal activity and metabolism continue into the postictal period, when impaired consciousness often persists in human TLE (Blumenfeld et al., 2004a,b). Also, the ictal neocortical slow oscillations we observed were very similar to slow wave activity seen in the same rats not during seizures, but during slow-wave sleep and under deep anesthesia.

While two different animal seizure models were used in this study, it is important to use caution in extending findings from anesthetized animals to awake-behaving animals and humans.
Although this is a notable limitation of the present study, it is also encouraging that electrographic rhythms during stimulated seizures in the anesthetized rats (Fig. 3, supplemental Fig. 3, available at www.jneurosci.org as supplemental material) closely resemble those observed during spontaneous limbic seizures in both awake rats (Figs. 1, 2) and in humans (Blumenfeld et al., 2004b). Also, neuroimaging and cerebrovascular decreases in the anesthetized rat neocortex during partial seizures bear similarity to neocortical SPECT decreases in humans during temporal lobe seizures (Blumenfeld et al., 2004a), further corroborating the usefulness of our lightly anesthetized rat model. Nevertheless, additional studies of behavior and physiology in awake behaving animals will be crucial to further confirm and extend these findings.

\section{Long-range network mechanisms of ictal neocortical}

slow activity

What are the long-range network mechanisms of ictal neocortical slow activity during partial seizures, and how do they differ from the mechanisms of seizure propagation during generalized seizures? The pathways responsible for seizure propagation in TLE have been previously studied, but remain poorly understood (Spencer et al., 1987; Wilson et al., 1990; Bertashius, 1991; Gloor 


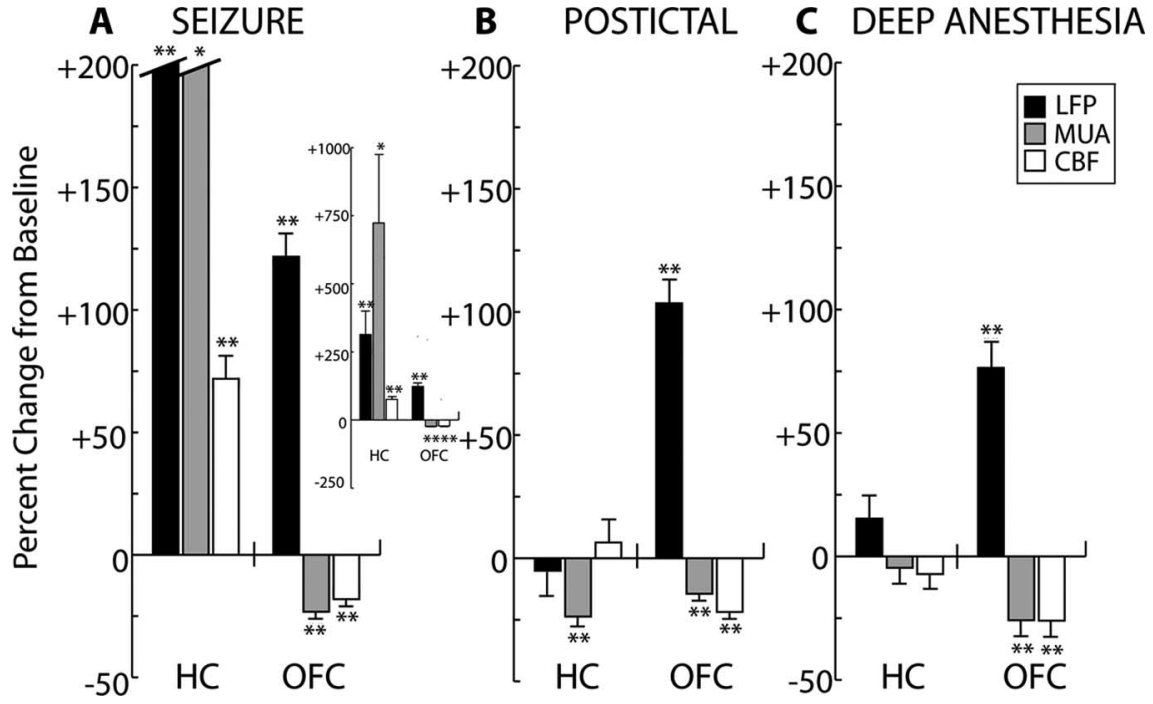

Figure 8. Depressed neuronal activity and CBF in the neocortex during partial limbic seizures resembles changes seen during deep anesthesia. LFP and MUA data are averaged across 17 recordings in 12 animals, whereas CBF data are from 15 seizures in 9 animals. Values are the mean percentage change ( \pm SEM) of $45 \mathrm{~s}$ data segments versus baseline, where baseline is defined as the last $45 \mathrm{~s}$ under light anesthesia before stimulus (Fig. 3). $\boldsymbol{A}$, During the first $45 \mathrm{~s}$ of seizure, in the hippocampus (HC) there is an increase in LFPs, MUA, and CBF compared with baseline. In the orbitofrontal cortex (OFC), there is an increase in LFPs but significant decreases in MUA and CBF. Hippocampal LFP and MUA columns are truncated, and shown in entirety in the inset. $\boldsymbol{B}$, During the first $45 \mathrm{~s}$ of the postictal period, there is significant depression of hippocampal MUA compared with baseline, and no significant change in LFPs or CBF. In the frontal cortex, LFP signal amplitude remains elevated and there is sustained suppression of both MUA and CBF. C, During deep anesthesia, no significant changes are seen in the hippocampus. Increased LFP activity and decreased MUA and CBF are observed in the orbitofrontal cortex, resembling frontal cortical activity seen during ictal and postictal slow activity $(\boldsymbol{A}, \boldsymbol{B}) .{ }^{*} p \leq 0.05,{ }^{* *} p \leq 0.01$, two-tailed $t$ test compared with baseline with Bonferroni's correction for multiple comparisons.

et al., 1993). It has been proposed that the orbitofrontal cortex might play an important role in cortical propagation of seizure activity (Lieb et al., 1991; Wilson and Engel, 1993) and has a high level of functional connectivity with the hippocampus in TLE (Wilson and Engel, 1993; Catenoix et al., 2005). Therefore, exploitation of normal hippocampal-neocortical circuits, which have vital roles in memory storage (Lavenex and Amaral, 2000; Thierry et al., 2000), may lead to abnormal cortical activity during hippocampal seizures. It is possible that the orbitofrontal cortex may act as gateway for neocortical propagation of seizure activity, exhibiting a depressed state of neuronal function during smaller complex partial seizures which is overwhelmed by exceedingly intense excitatory activity seen in secondarily generalized seizures.

Although a complete understanding of the circuitry involved in ictal neocortical slow waves requires additional study, our current results do provide preliminary insight into brain regions which may play an important role in this phenomenon. In addition to the hippocampus, regions which showed consistent BOLD activation and increased CBV during partial limbic seizures include the thalamus and the septal nuclei. Considering the similarities between ictal slow waves and slow oscillations observed during slow-wave sleep, it is possible that networks of thalamocortical and reticular thalamic neurons important for generating sleep rhythms may play a role in mediating this phenomenon (Steriade et al., 1991; Jones, 2002). The role of the thalamus in seizure propagation has been investigated previously (Blumenfeld and McCormick, 2000; Steriade, 2005), but thalamic activity during partial seizures with neocortical slow activity has not been characterized. We also observed considerable activations in the septal area during partial limbic seizures. Pre- vious work has shown that the septal nuclei play an important role in regulating normal hippocampal theta rhythms (Cavazos et al., 1997; Colom, 2006), and may provide an antiepileptogenic benefit by preventing hyperexcitable limbic states (Colom et al., 2006; Colom and GarridoSanabria, 2007). Possible effects of septal activity on the propagation of seizure activity out of the hippocampus deserve further investigation. Finally, it is important to note that up and down states of neuronal firing, similar to those seen during ictal neocortical slow waves or slow-wave sleep, have also been observed in prefrontal cortical slices deafferented from the rest of the brain (Timofeev et al., 2000; Compte et al., 2003; Shu et al., 2006). This raises interesting mechanistic questions about whether ictal neocortical slow waves represents an active inhibitory process, perhaps one which beneficially limits the spread of seizure activity, or more simply, a pathological state resulting from suspension of normal cortical excitation (Norden and Blumenfeld, 2002; Blumenfeld and Taylor, 2003).

\section{Conclusions}

In this study, we described abnormal neocortical function during partial limbic seizures in rats. Partial hippocampal seizures were associated with neocortical slow activity characterized by up and down states of neuronal firing, as well as decreased neuronal activity and fMRI signal in the frontal cortex. This activity differed markedly from seizure propagation observed in the neocortex during secondarily generalized seizures, which was characterized by fast polyspike activity, increased neuronal firing, and fMRI increases. In awake-behaving rats, behaviorally mild seizures were more likely than behaviorally severe seizures to be associated with ictal neocortical slow oscillations, which closely resembled cortical rhythms during slow-wave sleep or deep anesthesia. It is likely that in humans with TLE, ictal neocortical slow waves represent a pathophysiological consequence of limbic seizure activity, resulting in aberration of normal cortical function and loss of consciousness during complex partial seizures. Because impaired neocortical function and cognitive deficits have a large impact on quality of life in TLE patients, improved insight into the long-range effects of limbic seizures may lead to improved therapies geared at disrupting the cortical effects of partial epilepsy.

\section{References}

Achermann P, Borbély AA (1997) Low-frequency $(<1 \mathrm{~Hz})$ oscillations in the human sleep electroencephalogram. Neuroscience 81:213-222.

Angelucci A, Levitt JB, Lund JS (2002) Anatomical origins of the classical receptive field and modulatory surround field of single neurons in macaque visual cortical area V1. Prog Brain Res 136:373-388.

Bahar S, Suh M, Zhao M, Schwartz TH (2006) Intrinsic optical signal imaging of neocortical seizures: the "epileptic dip." Neuroreport 17:499-503.

Bazhenov M, Timofeev I, Steriade M, Sejnowski TJ (2002) Model of thalamocortical slow-wave sleep oscillations and transitions to activated States. J Neurosci 22:8691-8704.

Bertashius KM (1991) Propagation of human complex-partial seizures: a correlation analysis. Electroencephalogr Clin Neurophysiol 78:333-340. 
Blumenfeld H, McCormick DA (2000) Corticothalamic inputs control the pattern of activity generated in thalamocortical networks. J Neurosci 20:5153-5162.

Blumenfeld H, Taylor J (2003) Why do seizures cause loss of consciousness? Neuroscientist 9:301-310.

Blumenfeld H, McNally KA, Vanderhill SD, Paige AL, Chung R, Davis K, Norden AD, Stokking R, Studholme C, Novotny EJ Jr, Zubal IG, Spencer SS (2004a) Positive and negative network correlations in temporal lobe epilepsy. Cereb Cortex 14:892-902.

Blumenfeld H, Rivera M, McNally KA, Davis K, Spencer DD, Spencer SS (2004b) Ictal neocortical slowing in temporal lobe epilepsy. Neurology 63:1015-1021.

Bonilha L, Rorden C, Appenzeller S, Coan AC, Cendes F, Li LM (2006) Gray matter atrophy associated with duration of temporal lobe epilepsy. Neuroimage 32:1070-1079.

Bragin A, Engel J Jr, Wilson CL, Vizentin E, Mathern GW (1999) Electrophysiologic analysis of a chronic seizure model after unilateral hippocampal KA injection. Epilepsia 40:1210-1221.

Bragin A, Azizyan A, Almajano J, Wilson CL, Engel J Jr (2005) Analysis of chronic seizure onsets after intrahippocampal kainic acid injection in freely moving rats. Epilepsia 46:1592-1598.

Bragin A, Claeys P, Vonck K, Van Roost D, Wilson C, Boon P, Engel J Jr (2007) Analysis of initial slow waves (ISWs) at the seizure onset in patients with drug resistant temporal lobe epilepsy. Epilepsia 48:1883-1894.

Bruehl C, Kloiber O, Hossman KA, Dorn T, Witte OW (1995) Regional hypometabolism in an acute model of focal epileptic activity in the rat. Eur J Neurosci 7:192-197.

Brumberg JC, Pinto DJ, Simons DJ (1996) Spatial gradients and inhibitory summation in the rat whisker barrel system. J Neurophysiol 76:130-140.

Burchiel KJ, Stockard JJ, Calverley RK, Smith NT (1977) Relationship of pre- and postanesthetic EEG abnormalities to enflurane-induced seizure activity. Anesth Analg 56:509-514.

Catenoix H, Magnin M, Guénot M, Isnard J, Mauguière F, Ryvlin P (2005) Hippocampal-orbitofrontal connectivity in human: an electrical stimulation study. Clin Neurophysiol 116:1779-1784.

Cavalheiro EA, Leite JP, Bortolotto ZA, Turski WA, Ikonomidou C, Turski L (1991) Long-term effects of pilocarpine in rats: structural damage of the brain triggers kindling and spontaneous recurrent seizures. Epilepsia 32:778-782.

Cavazos JE, Wang CJ, Sitoh YY, Ng SE, Tien RD (1997) Anatomy and pathology of the septal region. Neuroimaging Clin N Am 7:67-78.

Chang DJ, Zubal IG, Gottschalk C, Necochea A, Stokking R, Studholme C, Corsi M, Slawski J, Spencer SS, Blumenfeld H (2002) Comparison of statistical parametric mapping and SPECT difference imaging in patients with temporal lobe epilepsy. Epilepsia 43:68-74.

Clark DL, Rosner BS (1973) Neurophysiologic effects of general anesthetics. I. The electroencephalogram and sensory evoked responses in man. Anesthesiology 38:564-582.

Collins RC (1978) Use of cortical circuits during focal penicillin seizures: an autoradiographic study with [14C] deoxyglucose. Brain Res 150:487-501.

Colom LV (2006) Septal networks: relevance to theta rhythm, epilepsy and Alzheimer's disease. J Neurochem 96:609-623.

Colom LV, Garrido-Sanabria E (2007) Modulation of normal and altered hippocampal excitability states by septal networks. J Neurosci Res 85:2839-2843.

Colom LV, García-Hernández A, Castañeda MT, Perez-Cordova MG, Garrido-Sanabria ER (2006) Septo-hippocampal networks in chronically epileptic rats: potential antiepileptic effects of theta rhythm generation. J Neurophysiol 95:3645-3653.

Compte A, Sanchez-Vives MV, McCormick DA, Wang XJ (2003) Cellular and network mechanisms of slow oscillatory activity $(<1 \mathrm{~Hz})$ and wave propagations in a cortical network model. J Neurophysiol 89:2707-2725.

Cowan RL, Wilson CJ (1994) Spontaneous firing patterns and axonal projections of single corticostriatal neurons in the rat medial agranular cortex. J Neurophysiol 71:17-32.

Crisler S, Morrissey MJ, Anch AM, Barnett DW (2008) Sleep-stage scoring in the rat using a support vector machine. J Neurosci Methods 168:524-534.

Diehl B, LaPresto E, Najm I, Raja S, Rona S, Babb T, Ying Z, Bingaman W, Lüders HO, Ruggieri P (2003) Neocortical temporal FDG-PET hypometabolism correlates with temporal lobe atrophy in hippocampal scle- rosis associated with microscopic cortical dysplasia. Epilepsia 44:559-564.

Ebersole JS, Pedley TA (2003) Current practice of clinical electroencephalography, Ed 3. Philadelphia: Lippincott, Williams and Wilkins.

Eisenschenk S, Gilmore RL, Cibula JE, Roper SN (2001) Lateralization of temporal lobe foci: depth versus subdural electrodes. Clin Neurophysiol 112:836-844.

Engel J Jr (1987) Outcome with respect to epileptic seizures. In: Surgical treatment of the epilepsies (Engel J Jr, ed), pp 553-571. New York: Raven.

Englot DJ, Blumenfeld H (2008) Functional MRI in basic epilepsy research. In: Encyclopedia of basic epilepsy research (Schwartzkroin PA, ed), in press.

Franaszczuk PJ, Bergey GK, Kamiński MJ (1994) Analysis of mesial temporal seizure onset and propagation using the directed transfer function method. Electroencephalogr Clin Neurophysiol 91:413-427.

Gloor P, Salanova V, Olivier A, Quesney LF (1993) The human dorsal hippocampal commissure. An anatomically identifiable and functional pathway. Brain 116:1249-1273.

Gottesmann C (1996) The transition from slow-wave sleep to paradoxical sleep: evolving facts and concepts of the neurophysiological processes underlying the intermediate stage of sleep. Neurosci Biobehav Rev 20:367-387.

Gruetter R (1993) Automatic, localized in vivo adjustment of all first- and second-order shim coils. Magn Reson Med 29:804-811.

Haider B, Duque A, Hasenstaub AR, McCormick DA (2006) Neocortical network activity in vivo is generated through a dynamic balance of excitation and inhibition. J Neurosci 26:4535-4545.

Handforth A, Ackermann RF (1988) Functional $\left[{ }^{14} \mathrm{C}\right] 2$-deoxyglucose mapping of progressive states of status epilepticus induced by amygdala stimulation in rat. Brain Res 460:96-102.

He J, Devonshire IM, Mayhew JE, Papadakis NG (2007) Simultaneous laser Doppler flowmetry and arterial spin labeling MRI for measurement of functional perfusion changes in the cortex. Neuroimage 34:1391-1404.

Helmstaedter C, Kockelmann E (2006) Cognitive outcomes in patients with chronic temporal lobe epilepsy. Epilepsia 47 [Suppl 2]:96-98.

Herman P, Sanganahalli BG, Hyder F (2008) Multi-modal measurements of blood plasma and red blood cell volumes during functional brain activation. J Cereb Blood Flow Metab, in press.

Hyder F, Kida I, Behar KL, Kennan RP, Maciejewski PK, Rothman DL (2001) Quantitative functional imaging of the brain: towards mapping neuronal activity by BOLD fMRI. NMR Biomed 14:413-431.

Hyder F, Kida I, Smith AJ, Blumenfeld H, Shulman RG, Rothman DL (2002) Quantitative fMRI of rat brain by multi-modal MRI and MRS measurements. International Congress Series 1235:57-71.

Jones EG (2002) Thalamic circuitry and thalamocortical synchrony. Philos Trans R Soc Lond B Biol Sci 357:1659-1673.

Kida I, Hyder F (2006) Physiology of functional magnetic resonance imaging: energetics and function. Methods Mol Med 124:175-195.

Kida I, Rothman DL, Hyder F (2007) Dynamics of changes in blood flow, volume, and oxygenation: implications for dynamic functional magnetic resonance imaging calibration. J Cereb Blood Flow Metab 27:690-696.

Laurent A, Arzimanoglou A (2006) Cognitive impairments in children with nonidiopathic temporal lobe epilepsy. Epilepsia 47 [Suppl 2]:99-102.

Lavenex P, Amaral DG (2000) Hippocampal-neocortical interaction: a hierarchy of associativity. Hippocampus 10:420-430.

Lieb JP, Dasheiff RM, Engel J Jr (1991) Role of the frontal lobes in the propagation of mesial temporal lobe seizures. Epilepsia 32:822-837.

Maandag NJ, Coman D, Sanganahalli BG, Herman P, Smith AJ, Blumenfeld H, Shulman RG, Hyder F (2007) Energetics of neuronal signaling and fMRI activity. Proc Natl Acad Sci U S A 104:20546-20551.

Mandeville JB, Marota JJ, Ayata C, Moskowitz MA, Weisskoff RM, Rosen BR (1999) MRI measurement of the temporal evolution of relative CMRO(2) during rat forepaw stimulation. Magn Reson Med 42:944-951.

Mayanagi Y, Watanabe E, Kaneko Y (1996) Mesial temporal lobe epilepsy: clinical features and seizure mechanism. Epilepsia 37 [Suppl 3]:57-60.

McCasland JS, Carvell GE, Simons DJ, Woolsey TA (1991) Functional asymmetries in the rodent barrel cortex. Somatosens Mot Res 8:111-116.

McCormick DA (2005) Neuronal networks: flip-flops in the brain. Curr Biol 15:R294-296.

Menzel C, Grünwald F, Klemm E, Ruhlmann J, Elger CE, Biersack HJ (1998) Inhibitory effects of mesial temporal partial seizures onto frontal neocortical structures. Acta Neurol Belg 98:327-331. 
Musizza B, Stefanovska A, McClintock PV, Palus M, Petrovcic J, Ribaric S, Bajrovic FF (2007) Interactions between cardiac, respiratory and EEGdelta oscillations in rats during anaesthesia. J Physiol 580:315-326.

Nelissen N, Van Paesschen W, Baete K, Van Laere K, Palmini A, Van Billoen H, Dupont P (2006) Correlations of interictal FDG-PET metabolism and ictal SPECT perfusion changes in human temporal lobe epilepsy with hippocampal sclerosis. Neuroimage 32:684-695.

Nersesyan H, Hyder F, Rothman DL, Blumenfeld H (2004) Dynamic fMRI and EEG recordings during spike-wave seizures and generalized tonicclonic seizures in WAG/Rij rats. J Cereb Blood Flow Metab 24:589-599.

Norden AD, Blumenfeld H (2002) The role of subcortical structures in human epilepsy. Epilepsy Behav 3:219-231.

Ogawa S, Menon RS, Tank DW, Kim SG, Merkle H, Ellermann JM, Ugurbil K (1993) Functional Brain mapping by blood oxygenation level-dependent contrast magnetic resonance imaging. Biophys J 64:803-812.

Paxinos G, Watson C (1998) The rat brain in stereotaxic coordinates. San Diego: Academic.

Plum F, Posner JB (1980) The diagnosis of stupor and coma. Philadelphia: Davis.

Prince DA, Wilder BJ (1967) Control mechanisms in cortical epileptogenic foci. "Surround" inhibition. Arch Neurol 16:194-202.

Rabinowicz AL, Salas E, Beserra F, Leiguarda RC, Vazquez SE (1997) Changes in regional cerebral blood flow beyond the temporal lobe in unilateral temporal lobe epilepsy. Epilepsia 38:1011-1014.

Racine RJ (1972) Modification of seizure activity by electrical stimulation. II. Motor seizure. Electroencephalogr Clin Neurophysiol 32:281-294.

Redecker C, Bruehl C, Hagemann G, Binus O, Witte OW (1997) Coupling of cortical and thalamic metabolism in experimentally induced visual and somatosensory focal epilepsy. Epilepsy Res 27:127-137.

Schridde U, Khubchandani M, Motelow JE, Sanganahalli BG, Hyder F, Blumenfeld H (2008) Negative BOLD with large increases in neuronal activity. Cereb Cortex 18:1814-1827.

Schwartz TH, Bonhoeffer T (2001) In vivo optical mapping of epileptic foci and surround inhibition in ferret cerebral cortex. Nat Med 7:1063-1067.

Shmuel A, Augath M, Oeltermann A, Logothetis NK (2006) Negative functional MRI response correlates with decreases in neuronal activity in monkey visual area V1. Nat Neurosci 9:569-577.

Shu Y, Hasenstaub A, Duque A, Yu Y, McCormick DA (2006) Modulation of intracortical synaptic potentials by presynaptic somatic membrane potential. Nature 441:761-765.

Spencer SS, Williamson PD, Spencer DD, Mattson RH (1987) Human hip- pocampal seizure spread studied by depth and subdural recording: the hippocampal commissure. Epilepsia 28:479-489.

Steriade M (2005) Sleep, epilepsy and thalamic reticular inhibitory neurons. Trends Neurosci 28:317-324.

Steriade M, Dossi RC, Paré D, Oakson G (1991) Fast oscillations (20-40 $\mathrm{Hz}$ ) in thalamocortical systems and their potentiation by mesopontine cholinergic nuclei in the cat. Proc Natl Acad Sci USA 88:4396-4400.

Steriade M, Nuñez A, Amzica F (1993) A novel slow ( $<1 \mathrm{~Hz}$ ) oscillation of neocortical neurons in vivo: depolarizing and hyperpolarizing components. J Neurosci 13:3252-3265.

Thierry AM, Gioanni Y, Dégénétais E, Glowinski J (2000) Hippocampoprefrontal cortex pathway: anatomical and electrophysiological characteristics. Hippocampus 10:411-419.

Timofeev I, Grenier F, Bazhenov M, Sejnowski TJ, Steriade M (2000) Origin of slow cortical oscillations in deafferented cortical slabs. Cereb Cortex 10:1185-1199.

Turski WA (2000) Pilocarpine-induced seizures in rodents-17 years on. Pol J Pharmacol 52:63-65.

Turski WA, Czuczwar SJ, Kleinrok Z, Turski L (1983) Cholinomimetics produce seizures and brain damage in rats. Experientia 39:1408-1411.

VanLandingham KE, Lothman EW (1991) Self-sustaining limbic status epilepticus. I. Acute and chronic cerebral metabolic studies: limbic hypermetabolism and neocortical hypometabolism. Neurology 41:1942-1949.

Van Paesschen W, Dupont P, Van Driel G, Van Billoen H, Maes A (2003) SPECT perfusion changes during complex partial seizures in patients with hippocampal sclerosis. Brain 126:1103-1111.

Velasco AL, Wilson CL, Babb TL, Engel J Jr (2000) Functional and anatomic correlates of two frequently observed temporal lobe seizure-onset patterns. Neural Plast 7:49-63.

Williamson PD, French JA, Thadani VM, Kim JH, Novelly RA, Spencer SS, Spencer DD, Mattson RH (1993) Characteristics of medial temporal lobe epilepsy: II. Interictal and ictal scalp electroencephalography, neuropsychological testing, neuroimaging, surgical results, and pathology. Ann Neurol 34:781-787.

Wilson CL, Engel J Jr (1993) Electrical stimulation of the human epileptic limbic cortex. Adv Neurol 63:103-113.

Wilson CL, Isokawa M, Babb TL, Crandall PH (1990) Functional connections in the human temporal lobe. I. Analysis of limbic system pathways using neuronal responses evoked by electrical stimulation. Exp Brain Res $82: 279-292$. 